\title{
A FIC-based stabilized finite element formulation for turbulent flows
}

\author{
Jordi Cotela-Dalmau, Riccardo Rossi, Eugenio Oñate \\ CIMNE - Centre Internacional de Metodes Numerics en Enginyeria, \\ Campus Nord UPC, c/Gran Capità s.n. 08034 Barcelona, Spain. \\ Universitat Politècnica de Catalunya, UPC Barcelona Tech, \\ c/Gran Capità s.n. 08034 Barcelona, Spain.
}

December 2, 2016

\begin{abstract}
We present a new stabilized finite element (FEM) formulation for incompressible flows based on the Finite Increment Calculus (FIC) framework [1]. In comparison to existing FIC approaches for fluids, this formulation involves a new term in the momentum equation, which introduces non-isotropic dissipation in the direction of velocity gradients. We also follow a new approach to the derivation of the stabilized mass equation, inspired by recent developments for quasi-incompressible flows [2]. The presented FIC-FEM formulation is used to simulate turbulent flows, using the dissipation introduced by the method to account for turbulent dissipation in the style of implicit large eddy simulation.
\end{abstract}

\section{Keywords}

Finite Increment Calculus; Turbulence modeling; Stabilized finite elements

\section{Introduction}

Simulation of turbulent flows represents one of the most challenging problems in computational fluid dynamics, due to the inherent difficulty in describing the flow and the wide range of spatial and temporal scales involved. In practice, the smallest flow features that can be captured in a numerical simulation are comparable in size to the mesh resolution and in duration to the time step. For turbulent problems, these are typically orders of magnitude larger than the smallest scales involved in the flow [3]. The effect of these unresolved motions on the large scale flow is typically incorporated in the simulation through the use of a turbulence model. Since, in turbulent flows, viscous dissipation predominantly occurs on the smallest scales, small scale motions tend to extract energy from the large scale flow. As a result, turbulence models are mostly dissipative in nature.

In finite element formulations for flow problems, numerical dissipation is often required to obtain a stable solution. The variational form of the Navier-Stokes equations has well-known numerical stability problems and a common way to prevent them is to modifying the equations to obtain a stable problem. Such stabilized formulations typically involve additional terms that are dissipative in nature. The interaction between numerically motivated dissipation from stabilized formulations and physically motivated dissipation from turbulence modeling requires careful consideration. This has driven research to verify that the results produced by stabilized finite element models of turbulent flow are physically relevant (e.g. $[4,5,6])$. In particular, in the context of variational multiscale (VMS) stabilized formulations $[7,8]$, an analogy has been proposed between the concept of scale separation and projection onto the finite element mesh that motivates VMS formulations and the spatial filters used in Large Eddy Simulation (LES) turbulence models [9, 10, 11]. This, providing a theoretical justification for the use of VMS stabilized formulations as the only source of dissipation in turbulent flow problems. This strategy is in the same vein as implicit LES formulations, such as monotonically-integrated LES (MILES) [12], which rely on numerically induced dissipation to achieve physically relevant results. This approach to turbulence modeling has been tested numerically in multiple studies $(e . g .[13,14,15,16])$.

Finite Increment Calculus (FIC), sometimes called Finite Calculus in short, is a stabilization technique based on writing the balance equations of the problem for an arbitrarily small, but finite-sized, domain [1, 17, 18]. The expression obtained in this way can be used as the starting point to obtain the weak form of the problem, in place of the original point-wise equation, and results in a variational problem with additional terms that have a stabilizing effect in the numerical solution. The FIC approach has been used in conjunction with the finite element method (FEM) to simulate incompressible flows at a range of Reynolds numbers [19, 20, 21] in 
the past. In this paper we present a new FIC-FEM formulation for the incompressible Navier-Stokes equations and explore the possibility of using it to simulate turbulent flows.

Compared to previous FIC-based approaches, the main novelties of the presented formulation are a new dissipative term in the momentum equation and a new approach to the derivation of the stabilized mass conservation equation. The new term in the momentum equation acts as a source of non-isotropic dissipation in the direction of velocity gradients. The stabilized mass equation is based on stating the second order FIC balance in space [22] for mass conservation and follows similar ideas to those recently used to derive a Lagrangian formulation for quasi-incompressible flows [2].

The paper is organized as follows: Section 2 presents the governing equations and the notation we use to describe them, Section 3 presents the derivation of the FIC-stabilized momentum equation and Section 4 does the same for mass conservation equation. Both equations are used in Section 5 to derive a stabilized FIC-FEM formulation, which is then applied to turbulent flow problems in Sections 6 and 7. Finally, Section 8 presents the main conclusions of the work.

\section{Governing equations}

The flow of an incompressible fluid within a domain of interest $\Omega$ is described by the Navier-Stokes equations, which state the balance of linear momentum and mass within $\Omega$ as

$$
\begin{aligned}
\rho \partial_{t} u_{i}+\rho u_{k} \frac{\partial u_{i}}{\partial x_{k}}-\frac{\partial \sigma_{i j}}{\partial x_{j}} & =f_{i} & & \text { in } \Omega \times[0, T) \quad i, j, k \in\left\{1, n_{d}\right\} \\
\frac{\partial u_{k}}{\partial x_{k}} & =0 & & \text { in } \Omega \times[0, T)
\end{aligned}
$$

where $\boldsymbol{u}=\left\{u_{i}\right\}$ represents the fluid velocity field, $\rho$ is the density and $\sigma=\left\{\sigma_{i j}\right\}$ is the stress tensor, $\boldsymbol{f}=\left\{f_{i}\right\}$ are the volume forces acting on the domain and $n_{d}$ represents the number of spatial dimensions $\left(n_{d}=3\right.$ for $3 D$ problems).

The problem is completed by an initial condition

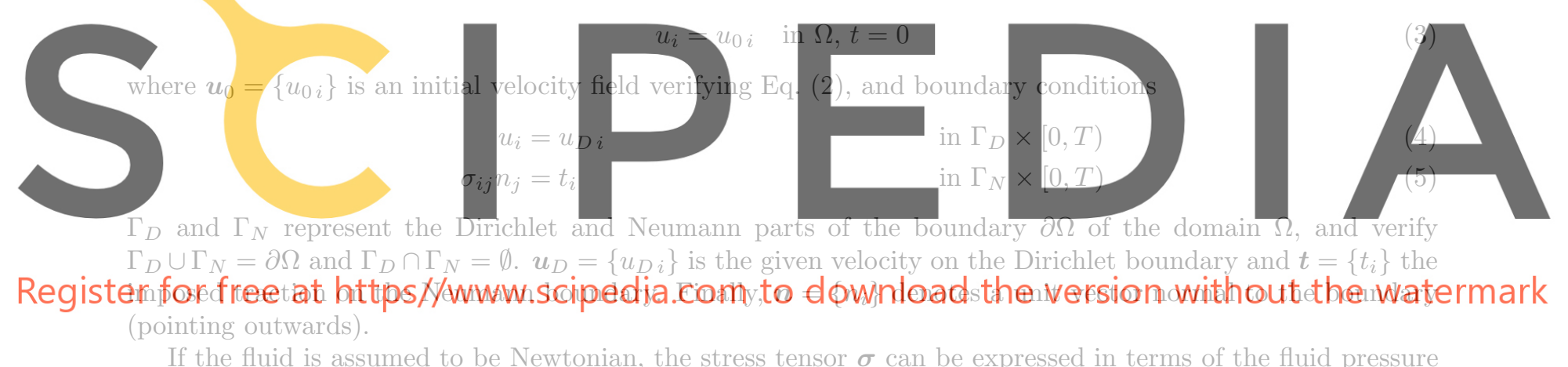

$p$ and the rate of strain tensor $\varepsilon$ as

$$
\begin{aligned}
\sigma_{i j} & =2 \mu\left(\varepsilon_{i j}-\frac{\varepsilon_{k k}}{3} \delta_{i j}\right)-p \delta_{i j} \\
\varepsilon_{i j} & =\frac{1}{2}\left(\frac{\partial u_{i}}{\partial x_{j}}+\frac{\partial u_{j}}{\partial x_{i}}\right)
\end{aligned}
$$

Eqs. (1) and (2), combined with initial and boundary conditions given by Eqs. (3)-(5), represent the complete Navier-Stokes problem. However, we use a modified version of the problem in the following. We replace the advective form of the convective term used in Eq. (1) with the skew-symmetric form, that is

$$
u_{k} \frac{\partial u_{i}}{\partial x_{k}}=\frac{1}{2}\left(u_{k} \frac{\partial u_{i}}{\partial x_{k}}+\frac{\partial}{\partial x_{k}}\left(u_{i} u_{k}\right)\right)
$$

Both forms of the convective term presented in Eq. (8) are identical when the differential form of the problem is considered, but yield different variational problems due to the fact that the velocity solution of the weak problem is only required to be divergence-free in an integral sense. As noted by Codina et al. in [23], only the skew-symmetric form of the convective term results in a variational problem that conserves kinetic energy.

It is well known that the variational form resulting from the Navier-Stokes problem has inherent stability issues, both due to the presence of the convective term and to the incompressibilty constraint that gives rise to the inf-sup or LBB condition on the acceptable interpolation spaces where the solution can be sought (see for example [24]). To solve these issues, we propose a stabilized formulation based on the FIC framework [17, 18]. We devote the following sections to present, in turn, the FIC balance equations for linear momentum and mass conservation that will be used to write a stabilized weak form of the problem. 


\section{Stabilized momentum equation}

We start by writing the linear momentum equation in residual form. Introducing Eq. (6) in Eq. (1) and using the skew-symmetric convective form of Eq. (8), the linear momentum residual is defined as

$$
r_{i}^{m}:=\rho \partial_{t} u_{i}+\rho\left(\frac{1}{2} u_{k} \frac{\partial u_{i}}{\partial x_{k}}+\frac{1}{2} \frac{\partial}{\partial x_{k}}\left(u_{i} u_{k}\right)\right)-2 \mu \frac{\partial}{\partial x_{j}}\left(\varepsilon_{i j}-\frac{\varepsilon_{k k}}{3} \delta_{i j}\right)+\frac{\partial p}{\partial x_{i}}-f_{i}
$$

The first order FIC balance equation in space [18] can be used to obtain a stabilized formulation for the momentum equation. For a vector equation such as Eq. (9), this results in [17]

$$
r_{i}^{m}-\frac{h_{i j}}{2} \frac{\partial r_{i}^{m}}{\partial x_{j}}=0 \quad i, j \in\left\{1, n_{d}\right\} \quad \text { No sum on } i \text {. }
$$

where $h_{i j}$ represents a tensor of characteristic lengths, which is related to the dimensions of the finite-sized domain used to write the FIC balance equation. Specifically, for each component of the momentum residual $r_{i}^{m}$, the $i$-th row of $h_{i j}$ defines the characteristic lengths along coordinate directions $j$ of the domain used to write the $i$-th FIC balance equation. Note that, if the same domain is used to obtain a FIC balance for every component of the linear momentum equation, the rows of $h_{i j}$ will be identical. In this particular case, index $i$ can be dropped and we can speak of a vector of characteristic lengths $\boldsymbol{h}=\left\{h_{j}\right\}$, which corresponds to the notation used in $[1,17]$.

We consider different possibilities to design $h_{i j}$. The first one is to define the characteristic lengths based on the mesh size along the streamlines of the flow. This results in a method similar to the SUPG formulation [25]. A second option is to base the characteristic length on the size of the element along the direction of the gradient of velocity. This acts as a source of additional diffusion, although the resulting formulation is not stable by itself. Finally, we will also consider the possibility of combining both approaches.

\subsection{Streamline diffusion formulation}

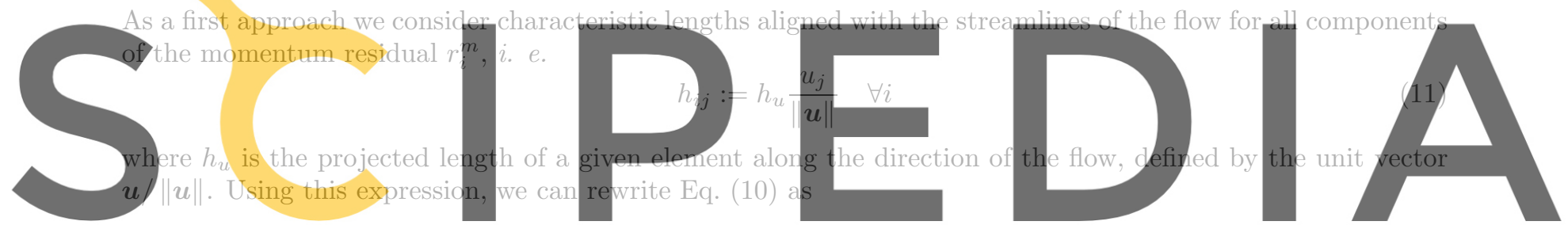

Register for free at https//www.scipedia.com to download the version without the watermark

Eq. (12) can be used as the starting point to write a stabilized formulation for the momentum equation. Multiplying by a test function $w_{i}$ and integrating over the fluid domain we obtain

$$
\int_{\Omega}\left(w_{i} r_{i}^{m}-w_{i} \frac{h_{u}}{2\|\boldsymbol{u}\|} u_{k} \frac{\partial r_{i}^{m}}{\partial x_{k}}\right) \mathrm{d} \Omega=0
$$

It is convenient to integrate by parts the second term in the left hand side of Eq. (13). Note that, as the length $h_{u}$ will be defined as a constant quantity within each element, the boundary integral that appears in the integration by parts should be understood as an integral over all elemental boundaries. This operation produces

$$
\int_{\Omega} w_{i} r_{i}^{m} \mathrm{~d} \Omega+\int_{\Omega} \frac{h_{u}}{2\|\boldsymbol{u}\|} u_{k} \frac{\partial w_{i}}{\partial x_{k}} r_{i}^{m} \mathrm{~d} \Omega-\sum_{(e)} \int_{\Gamma_{e}} \frac{h_{u}}{2\|\boldsymbol{u}\|} w_{i}\left(u_{k} n_{k}\right) r_{i}^{m} \mathrm{~d} \Gamma=0
$$

We have neglected the elemental boundary integrals that appear in Eq. (14) in the present work, which can be understood as assuming that $r_{i}^{m}$ vanishes on the element boundaries. At this point, we introduce in Eq. (14) the definition of the residual, given by Eq. (9), and its gradient in Eq. (14). Integrating by parts the viscous and pressure terms within the residual in the first integral of Eq. (14) gives

$$
\begin{gathered}
\int_{\Omega} w_{i} \rho\left(\partial_{t} u_{i}+\frac{1}{2} u_{k} \frac{\partial u_{i}}{\partial x_{k}}\right) \mathrm{d} \Omega-\int_{\Omega} \frac{1}{2} u_{k} \frac{\partial w_{i}}{\partial x_{k}} \rho u_{i} \mathrm{~d} \Omega+\int_{\Omega} 2 \mu \frac{\partial w_{i}}{\partial x_{j}}\left(\varepsilon_{i j}-\frac{\varepsilon_{k k}}{3} \delta_{i j}\right) \mathrm{d} \Omega-\int_{\Omega} \frac{\partial w_{i}}{\partial x_{i}} p \mathrm{~d} \Omega \\
+\int_{\Omega} \frac{h_{u}}{2\|\boldsymbol{u}\|} u_{k} \frac{\partial w_{i}}{\partial x_{k}}\left(\rho \partial_{t} u_{i}+\rho\left(\frac{1}{2} u_{k} \frac{\partial u_{i}}{\partial x_{k}}+\frac{1}{2} \frac{\partial}{\partial x_{k}}\left(u_{i} u_{k}\right)\right)+\frac{\partial p}{\partial x_{i}}-2 \mu \frac{\partial}{\partial x_{j}}\left(\varepsilon_{i j}-\frac{\varepsilon_{k k}}{3} \delta_{i j}\right)-f_{i}\right) \mathrm{d} \Omega= \\
\int_{\Omega} w_{i} f_{i} \mathrm{~d} \Omega+\int_{\Gamma_{N}} w_{i} t_{i} \mathrm{~d} \Gamma-\int_{\Gamma_{N}} \frac{1}{2} \rho w_{i}\left(u_{k} n_{k}\right) u_{i} \mathrm{~d} \Gamma
\end{gathered}
$$


where $t_{i}$ represents the $i$-th component of the tractions imposed on the Neumann boundary $\Gamma_{N}$.

Note that, although Eq. (15) has been developed using a FIC based approach, the final expression is analogous to a SUPG stabilized formulation, with $h_{u} / 2\|\boldsymbol{u}\|$ (which has dimensions of time) playing the role of the SUPG stabilization parameter $\tau[25]$.

Next, we need to provide a definition for $h_{u}$ to complete the formulation. In the present approach, we calculate the characteristic element length using the size of the element in the direction of velocity $\boldsymbol{u}$. Defining the unit vector in the direction of velocity as $\boldsymbol{e}_{u}$ and representing the element edge joining nodes $a$ and $b$ with the vector $\boldsymbol{l}_{a b}$, the element length is given by

$$
h_{u}=\max _{\text {edges }}\left\{\boldsymbol{e}_{u} \cdot \boldsymbol{l}_{a b}\right\} \quad \text { with } \quad \boldsymbol{e}_{u}=\frac{\boldsymbol{u}}{\|\boldsymbol{u}\|}
$$

This is shown graphically for triangles and quadrilaterals in Fig. 1, but the same procedure is applied in $3 D$ to obtain the elemental length using the edges of tetrahedra and hexahedra.
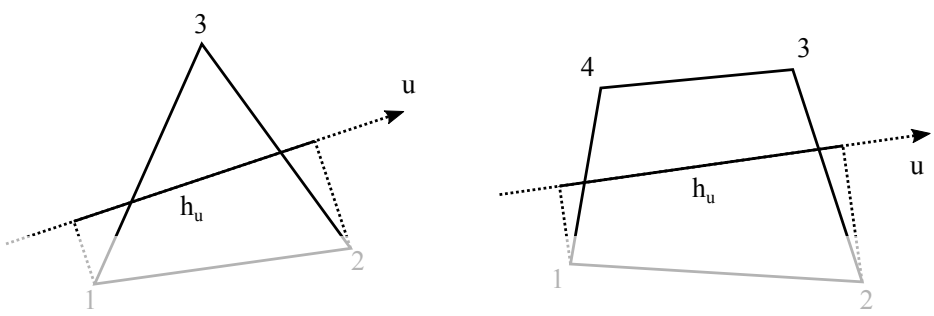

Figure 1: Definition of the element lenght $h_{u}$ for triangles and quadrilaterals.

In practice, Eq. (16) is evaluated at the integration points of each element. In the case that velocity is (close to) zero on a given point for a given time step, $e_{u}$ is undefined and this expression cannot be used. If this happens, an average element length can be used instead. The characteristic length we use in this case is the
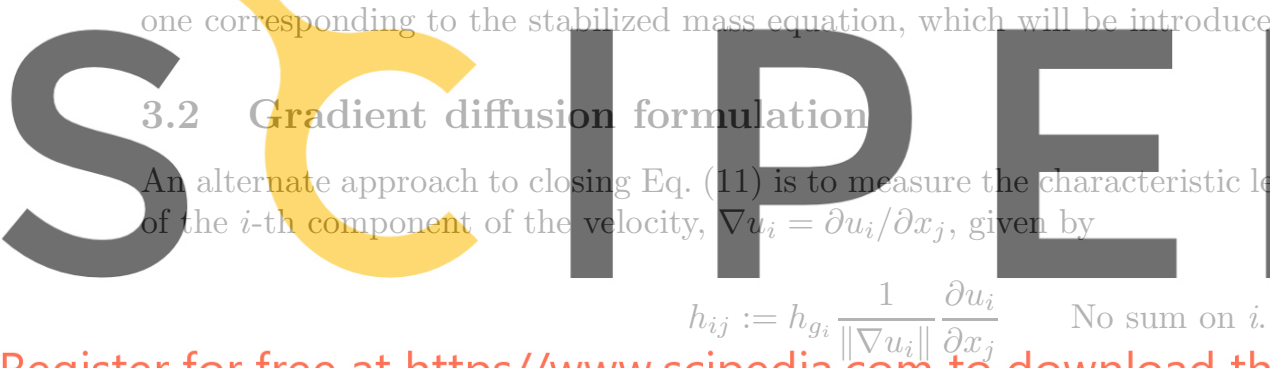

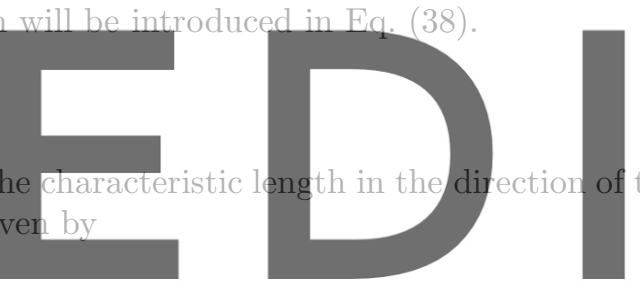

Register for free at https//www.scipedia.com to download the version without the watermark which, as before, can be used to write a FIC balance statement for each component of the momentum equation, resulting in

$$
r_{i}^{m}-\frac{h_{g_{i}}}{2\left\|\nabla u_{i}\right\|} \frac{\partial u_{i}}{\partial x_{j}} \frac{\partial r_{i}^{m}}{\partial x_{j}} \quad \text { No sum on } i .
$$

We can obtain a variational form of the FIC momentum balance equation defined by Eq. (18) by following the same procedure as for the streamline formulation in Section 3.1. Multiplying Eq. (18) by a test function $w_{i}$ and integrating over the fluid domain we obtain

$$
\int_{\Omega} w_{i} r_{i}^{m} \mathrm{~d} \Omega-\int_{\Omega} w_{i} \frac{h_{g_{i}}}{2\left\|\nabla u_{i}\right\|} \frac{\partial u_{i}}{\partial x_{j}} \frac{\partial r_{i}^{m}}{\partial x_{j}} \mathrm{~d} \Omega=0
$$

The first integral in Eq. (19) is identical to the first term of Eq. (14) and can be developed as in the previous section. We direct our attention towards the second term in Eq. (19), which can be integrated by parts as follows

$$
\begin{array}{r}
-\int_{\Omega} w_{i} \frac{h_{g_{i}}}{2\left\|\nabla u_{i}\right\|} \frac{\partial u_{i}}{\partial x_{j}} \frac{\partial r_{i}^{m}}{\partial x_{j}} \mathrm{~d} \Omega= \\
\int_{\Omega} \frac{\partial}{\partial x_{j}}\left(w_{i} \frac{h_{g_{i}}}{2\left\|\nabla u_{i}\right\|} \frac{\partial u_{i}}{\partial x_{j}}\right) r_{i}^{m}-\int_{\Omega} \frac{\partial}{\partial x_{j}}\left(w_{i} \frac{h_{g_{i}}}{2\left\|\nabla u_{i}\right\|} \frac{\partial u_{i}}{\partial x_{j}} r_{i}^{m}\right) \mathrm{d} \Omega= \\
\int_{\Omega} \frac{\partial w_{i}}{\partial x_{j}} \frac{h_{g_{i}}}{2\left\|\nabla u_{i}\right\|} \frac{\partial u_{i}}{\partial x_{j}} r_{i}^{m} \mathrm{~d} \Omega+\int_{\Omega} w_{i} \frac{\partial}{\partial x_{j}}\left(\frac{h_{g_{i}}}{2\left\|\nabla u_{i}\right\|} \frac{\partial u_{i}}{\partial x_{j}}\right) r_{i}^{m} \mathrm{~d} \Omega-\int_{\Omega} \frac{\partial}{\partial x_{j}}\left(w_{i} \frac{h_{g_{i}}}{2\left\|\nabla u_{i}\right\|} \frac{\partial u_{i}}{\partial x_{j}} r_{i}^{m}\right) \mathrm{d} \Omega
\end{array}
$$

From the three terms in the last equality of Eq. (20), only the first is retained. The second one is neglected as it involves either spatial derivatives of the characteristic length or second derivatives of velocity. The last 
term can be transformed into a boundary integral using the divergence theorem, and is dropped for the same reasons we neglected the boundary terms in the streamline formulation. Finally, it is convenient to rewrite the remaining term as

$$
\int_{\Omega} \frac{\partial w_{i}}{\partial x_{j}}\left(\frac{h_{g_{i}} r_{i}^{m}}{2\left\|\nabla u_{i}\right\|} \delta_{i k}\right) \frac{\partial u_{k}}{\partial x_{j}} \mathrm{~d} \Omega
$$

If we choose the characteristic length such that $h_{g_{i}} r_{i}^{m}>0$, Eq. (21) describes the discrete version of a nonisotropic Laplacian, where the diffusivity for each coordinate direction is different. The diffusivity coefficient in this case is proportional to the magnitude of the finite element residual on each coordinate direction and exhibits a similar structure to that of a shock-capturing formulation, such as the ones analyzed in [26]. The numerical diffusion added on each direction is defined by the diagonal tensor $D_{i j}^{g}$ as

$$
D_{i j}^{g}=\frac{h_{g_{i}} r_{i}^{m}}{2\left\|\nabla u_{i}\right\|} \delta_{i j} \quad \text { No sum on } i \text {. }
$$

Returning to Eq. (19), this reasoning allows us to rewrite the weak form for the gradient diffusion formulation as

$$
\int_{\Omega} w_{i} r_{i}^{m} \mathrm{~d} \Omega+\int_{\Omega} \frac{\partial w_{i}}{\partial x_{j}} D_{i k}^{g} \frac{\partial u_{k}}{\partial x_{j}} \mathrm{~d} \Omega=0
$$

As in the streamline diffusion case, we need to provide a definition for the characteristic lengths to close the formulation. We follow an analogous procedure to do so, now using the gradient of the $i$-th component of velocity to define the direction of projection. The characteristic element length for the $i$-th coordinate direction is therefore defined as

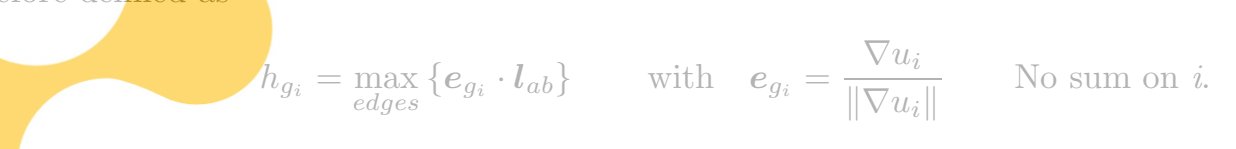

It must be noted that the formulation of Eq. (23) by itself is not sufficient to stabilize convection-dominated flows in general. A possible solution, which is presented in the following section, is to combine the gradient-
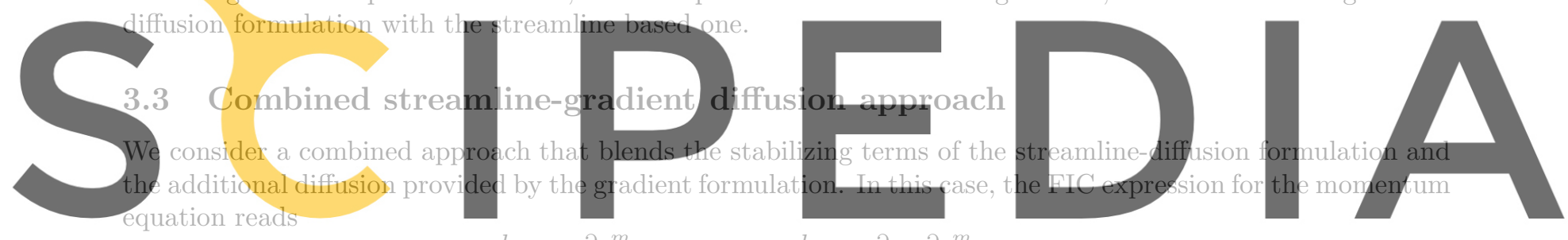

Register for free at https//wwwtscipedia.com to blow

where $\beta_{i} \in[0,1]$ is a combination parameter, which, in principle, can be different for each component of the momentum equation.

The combined variational formulation can be derived following the same steps as each of its components, which has been presented in the previous pages. Therefore, the final expression for the weak form, obtained by multiplying Eq. (15) by $\beta_{i}$ and Eq. (23) by $1-\beta_{i}$ and adding the result, is given as

$$
\begin{aligned}
\int_{\Omega} w_{i} \rho\left(\partial_{t} u_{i}\right. & \left.+\frac{1}{2} u_{k} \frac{\partial u_{i}}{\partial x_{k}}\right) \mathrm{d} \Omega-\int_{\Omega} \frac{1}{2} u_{k} \frac{\partial w_{i}}{\partial x_{k}} \rho u_{i} \mathrm{~d} \Omega+\int_{\Omega} 2 \mu \frac{\partial w_{i}}{\partial x_{j}}\left(\varepsilon_{i j}-\frac{\varepsilon_{k k}}{3} \delta_{i j}\right) \mathrm{d} \Omega-\int_{\Omega} \frac{\partial w_{i}}{\partial x_{i}} p \mathrm{~d} \Omega \\
+\int_{\Omega} \beta_{i} \frac{h_{u}}{2\|\boldsymbol{u}\|} u_{k} & \frac{\partial w_{i}}{\partial x_{k}}\left(\rho \partial_{t} u_{i}+\rho\left(\frac{1}{2} u_{k} \frac{\partial u_{i}}{\partial x_{k}}+\frac{1}{2} \frac{\partial}{\partial x_{k}}\left(u_{i} u_{k}\right)\right)+\frac{\partial p}{\partial x_{i}}-2 \mu \frac{\partial}{\partial x_{j}}\left(\varepsilon_{i j}-\frac{\varepsilon_{k k}}{3} \delta_{i j}\right)-f_{i}\right) \mathrm{d} \Omega \\
& +\int_{\Omega} \frac{\partial w_{i}}{\partial x_{j}}\left(1-\beta_{i}\right) D_{i k}^{g} \frac{\partial u_{k}}{\partial x_{j}} \mathrm{~d} \Omega=\int_{\Omega} w_{i} f_{i} \mathrm{~d} \Omega+\int_{\Gamma_{N}} w_{i} t_{i} \mathrm{~d} \Gamma-\int_{\Gamma_{N}} \frac{1}{2} \rho w_{i}\left(u_{k} n_{k}\right) u_{i} \mathrm{~d} \Gamma
\end{aligned}
$$

with $D_{i k}^{g}$ as defined by Eq. (22).

In principle, the combination parameter $\beta_{i}$ can take any value in the range $\beta_{i} \in[0,1]$. The limit case $\beta_{i}=1$ results in the classical FIC streamline formulation for the momentum equations, used for example in [18] or [20]. This formulation is very close to the SUPG stabilization procedure, but uses the stabilization parameter of Eq. (13), derived from FIC principles. On the other end of the range, $\beta_{i}=0$ implies using the gradient diffusion term exclusively and results in a formulation that is not numerically stable for convection-dominated problems. In the present work, we have experimented using a constant value $\beta_{i}=\beta$ for all components of the momentum equation. Numerical results have evidenced that values of $\beta \geq 0.5$ are typically needed for the problem to be stable for all flow regimes, while values of $\beta$ in the range $0.7 \sim 0.9$ typically give the best results. 
In addition to defining the value of $\beta_{i}$ as a fixed quantity for the entire simulation, we have also experimented with the possibility of using the local features of the flow to derive a value for $\beta_{i}$. In these simulations we have set a local value for $\beta_{i}$ depending on the directions of velocity and its gradient:

$$
\beta_{i}=\max \left\{1-\frac{u_{k}\left(\partial u_{i} / \partial x_{k}\right)}{\|\boldsymbol{u}\|\left\|\nabla u_{i}\right\|}, \beta_{m}\right\} \quad \text { No sum on } i .
$$

where $\beta_{m}$ is a minimum value to prevent the loss of stability if the velocity becomes parallel to $\nabla u_{i}$ in some point. Note that the value of $\beta_{i}$ that is obtained using Eq. (27) is different for each coordinate direction $i$. This means that, if this expression is used, both the streamline terms and the gradient terms in Eq. (26) are non-isotropic.

\section{Stabilized mass balance equation}

We follow the approach presented in $[22,21]$ to obtain a stabilized formulation for the mass balance equation given by Eq. (2). A similiar strategy was used in [2] to for a quasi-incompressible fluid formulation. We introduce the following notation for the mass balance residual:

$$
r^{c}:=\frac{\partial u_{k}}{\partial x_{k}}=\varepsilon_{k k}
$$

which can be used to derive the second order FIC balance in space as (see [22])

$$
r^{c}+\frac{h_{j}^{2}}{12} \frac{\partial^{2} r^{c}}{\partial x_{j}^{2}}=\varepsilon_{k k}+\frac{h_{j}^{2}}{12} \frac{\partial^{2} \varepsilon_{k k}}{\partial x_{j}^{2}}=0
$$

The expression of second order mass balance was originally introduced to obtain a stabilized formulation for incompressible flows in [22], where it was derived by expressing the balance of mass within a rectangular domain. This reference shows that Eq. (29) can be obtained by writing the velocities along the boundaries of the rectangle as a Taylor series expansion of the velocity on its center and retaining terms up to third order.

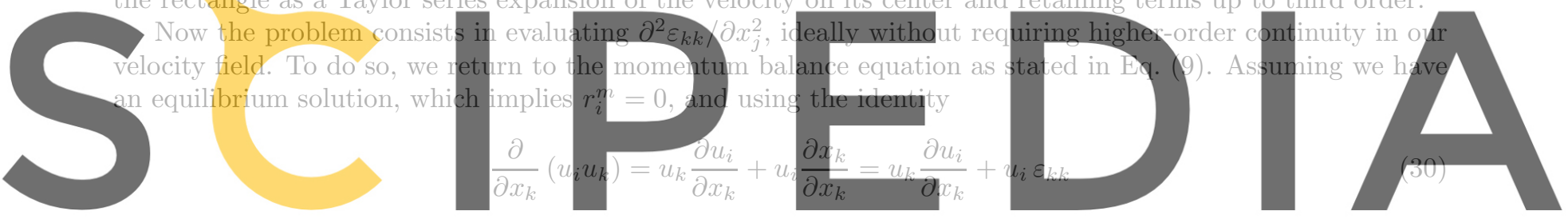

we can rearrange the terms in the momentum balance equation to read

\section{Register for free at https//Www.}

Grouping all terms involving $\varepsilon_{k k}$ we obtain

$$
-\frac{\rho u_{i}}{2} \varepsilon_{k k}-\frac{2 \mu}{3} \frac{\partial \varepsilon_{k k}}{\partial x_{i}}=\rho \partial_{t} u_{i}+\rho u_{k} \frac{\partial u_{i}}{\partial x_{k}}-2 \mu \frac{\partial \varepsilon_{i j}}{\partial x_{j}}+\frac{\partial p}{\partial x_{i}}-f_{i}=\hat{r}_{i}^{m}
$$

where we introduced the notation of $\hat{r}_{i}^{m}$ for the right hand side of Eq. (32) for compactness. At this point it is helpful to write the first order FIC balance for the continuity equation [17]

$$
\varepsilon_{k k}-\frac{h_{i}}{2} \frac{\partial \varepsilon_{k k}}{\partial x_{i}}=0
$$

and use it to express $\varepsilon_{k k}$ in terms of its derivative in Eq. (32). This gives

$$
-\left(\frac{\rho u_{j} h_{j}}{4}+\frac{2 \mu}{3}\right) \frac{\partial \varepsilon_{k k}}{\partial x_{i}}=\hat{r}_{i}^{m}
$$

Next we introduce Eq. (34) in Eq. (29) to write

$$
r^{c}+\frac{h_{i}^{2}}{12} \frac{\partial}{\partial x_{i}}\left(\frac{\partial \varepsilon_{k k}}{\partial x_{i}}\right)=r^{c}-\frac{h_{i}^{2}}{12} \frac{\partial}{\partial x_{i}}\left(\left(\frac{\rho u_{j} h_{j}}{4}+\frac{2 \mu}{3}\right)^{-1} \hat{r}_{i}^{m}\right)=0
$$

Neglecting the spatial variation of the product $h_{j} u_{j}$, we take the coefficient that multiplies $\hat{r}_{i}^{m}$ out of the derivative, obtaining

$$
r^{c}-\frac{h_{i}^{2}}{12}\left(\frac{\rho u_{j} h_{j}}{4}+\frac{2 \mu}{3}\right)^{-1} \frac{\partial \hat{r}_{i}^{m}}{\partial x_{i}}=0
$$


Eq. (36) expresses the basic FIC mass balance statement used in the present work. In our computation we use however a slightly modified version of Eq. (36), following the ideas of [2]

$$
r^{c}-\tau_{c} \frac{\partial \hat{r}_{i}^{m}}{\partial x_{i}}=0
$$

where $\tau_{c}$ is an isotropic stabilization parameter defined as

$$
\tau_{c}:=\left(\frac{3 \rho\|\boldsymbol{u}\|}{h}+\frac{8 \mu}{h^{2}}\right)^{-1}
$$

In the expression of $\tau_{c}$ we use the norm of the velocity and the average element length $h$, which is calculated as the square root of the elemental area in $2 D$ or the cubic root of the elemental volume in $3 D$.

Next we multiply Eq. (37) by a test function $q$ and integrate over the fluid domain $\Omega$ to obtain the weak form of the FIC mass balance equation

$$
\int_{\Omega} q r^{c} \mathrm{~d} \Omega-\int_{\Omega} q \tau_{c} \frac{\partial}{\partial x_{i}}\left(\rho \partial_{t} u_{i}+\rho u_{k} \frac{\partial u_{i}}{\partial x_{k}}-2 \mu \frac{\partial}{\partial x_{j}} \varepsilon_{i j}+\frac{\partial p}{\partial x_{i}}-f_{i}\right) \mathrm{d} \Omega=0
$$

It is convenient to integrate by parts the second integral in Eq. (39) to reduce the order of the derivatives involved. As in the momentum equation, the boundary terms resulting from this operation are neglected in the present work, obtaining the expression

$$
\int_{\Omega} q \frac{\partial u_{i}}{\partial x_{i}} \mathrm{~d} \Omega+\int_{\Omega} \frac{\partial q}{\partial x_{i}} \tau_{c}\left(\rho \partial_{t} u_{i}+\rho u_{k} \frac{\partial u_{i}}{\partial x_{k}}-2 \mu \frac{\partial}{\partial x_{j}} \varepsilon_{i j}+\frac{\partial p}{\partial x_{i}}-f_{i}\right) \mathrm{d} \Omega=0
$$

Eq. (40) represents a FIC-based stabilized formulation for the continuity equation, similar to that obtained in Galerkin-Least squares (GLS) formulations [27]. Note that the stabilization parameter $\tau_{c}$, defined in Eq. (38), has the same structure as the classical SUPG or GLS characteristic time $\tau$.

In addition to the formulation given by Eq. (40), we have also tested a variant involving the projection of $\hat{r}_{i}^{m}$ onto the finite element mesh. Consider the following modified version of Eq. (37)

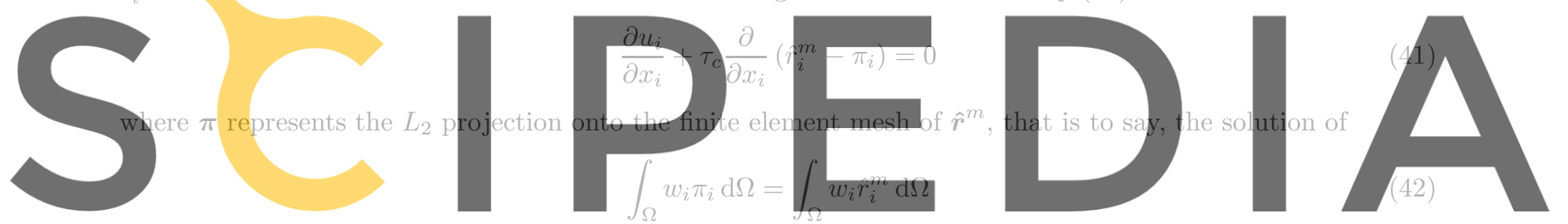

This formulation results in the following weak form, again neglecting boundary terms, which substitutes Eq. (40). Register for free at https//www.scipedia.com to download the version without the watermark

$$
\int_{\Omega} q \frac{\partial u_{i}}{\partial x_{i}} \mathrm{~d} \Omega+\int_{\Omega} \frac{\partial q}{\partial x_{i}} \tau_{c}\left(\rho \partial_{t} u_{i}+\rho u_{k} \frac{\partial u_{i}}{\partial x_{k}}-2 \mu \frac{\partial}{\partial x_{j}} \varepsilon_{i j}+\frac{\partial p}{\partial x_{i}}-f_{i}+\pi_{i}\right) \mathrm{d} \Omega=0
$$

We use Eq. (43) as the reference formulation in the following, with the understanding that any terms involving $\pi_{i}$ can be dropped to recover the formulation without projections.

\section{$5 \quad$ Finite element formulation}

Combining the stabilized momentum equation given by Eq. (26) and that of Eq. (43) for the continuity equation we write the complete stabilized weak form of the problem, which can be used to obtain a finite element formulation.

In the present work we restrict ourselves to linear finite elements, using triangular and quadrilateral elements in $2 D$ or tetrahedra and hexahedra in $3 D$. This means that all terms involving second derivatives of velocity in Eqs. (26) and (43) can be neglected, as they are identically zero when using linear interpolations. The full formulation, without second order terms, is given by

\section{Momentum}

$$
\begin{gathered}
\int_{\Omega} w_{i} \rho\left(\partial_{t} u_{i}+\frac{1}{2} u_{k} \frac{\partial u_{i}}{\partial x_{k}}\right) \mathrm{d} \Omega-\int_{\Omega} \frac{1}{2} u_{k} \frac{\partial w_{i}}{\partial x_{k}} \rho u_{i} \mathrm{~d} \Omega+\int_{\Omega} 2 \mu \frac{\partial w_{i}}{\partial x_{j}}\left(\varepsilon_{i j}-\frac{\varepsilon_{k k}}{3} \delta_{i j}\right) \mathrm{d} \Omega-\int_{\Omega} \frac{\partial w_{i}}{\partial x_{i}} p \mathrm{~d} \Omega \\
+\int_{\Omega} \beta_{i} \frac{h_{u}}{2\|\boldsymbol{u}\|} u_{k} \frac{\partial w_{i}}{\partial x_{k}}\left(\rho \partial_{t} u_{i}+\rho\left(\frac{1}{2} u_{k} \frac{\partial u_{i}}{\partial x_{k}}+\frac{1}{2} \frac{\partial}{\partial x_{k}}\left(u_{i} u_{k}\right)\right) \frac{\partial p}{\partial x_{i}}-f_{i}\right) \mathrm{d} \Omega+\int_{\Omega} \frac{\partial w_{i}}{\partial x_{j}}\left(1-\beta_{i}\right) D_{i k}^{g} \frac{\partial u_{k}}{\partial x_{j}} \mathrm{~d} \Omega= \\
\int_{\Omega} w_{i} f_{i} \mathrm{~d} \Omega+\int_{\Gamma_{N}} w_{i} t_{i} \mathrm{~d} \Gamma-\int_{\Gamma_{N}} \frac{1}{2} \rho w_{i}\left(u_{k} n_{k}\right) u_{i} \mathrm{~d} \Gamma
\end{gathered}
$$




$$
\int_{\Omega} q \frac{\partial u_{i}}{\partial x_{i}} \mathrm{~d} \Omega+\int_{\Omega} \frac{\partial q}{\partial x_{i}} \tau_{c}\left(\rho \partial_{t} u_{i}+\rho u_{k} \frac{\partial u_{i}}{\partial x_{k}}+\frac{\partial p}{\partial x_{i}}-f_{i}+\pi_{i}\right) \mathrm{d} \Omega=0
$$

with

$$
D_{i k}^{g}=\frac{h_{g_{i}} r_{i}^{m}}{2\left\|\nabla u_{i}\right\|} \delta_{i k} \quad \tau_{c}=\left(\frac{3 \rho\|\boldsymbol{u}\|}{h}+\frac{8 \mu}{h^{2}}\right)^{-1} \quad \beta_{i} \in[0,1]
$$

\subsection{Spatial discretization}

We introduce a finite element discretization $\Omega_{h}$ of the problem domain $\Omega$. The problem variables $\boldsymbol{u}$ and $p$ can be then approximated with the usual finite element interpolation as

$$
u_{h i}=\sum_{a}^{n_{n}} N_{a}(\boldsymbol{x}) u_{a i} \quad p_{h}=\sum_{a}^{n_{n}} N_{a}(\boldsymbol{x}) p_{a}
$$

where $n_{n}$ represents the number of nodes in the finite element mesh, $\boldsymbol{u}_{a}$ and $p_{a}$ are the variables evaluated at node $a$ and $N_{a}(\boldsymbol{x})$ is the standard linear finite element function associated to node $a$. Furthermore, we introduce the notation $\boldsymbol{U}, \dot{\boldsymbol{U}}$ and $\boldsymbol{P}$ to indicate the vectors of nodal values for velocity, acceleration and pressure, respectively. Introducing the finite element interpolation of Eq. (46) in Fqs. (44) and (45) and taking in turn each each nodal shape function $N_{a}$ as test functions $w_{i}, q$, we obtain a system of $n_{n}$ equations that can be expressed in matrix form as

$$
\left[\begin{array}{cc}
\boldsymbol{M}+\boldsymbol{M}_{K} & 0 \\
\boldsymbol{M}_{D} & 0
\end{array}\right]\left[\begin{array}{c}
\dot{U} \\
0
\end{array}\right]+\left[\begin{array}{cc}
\boldsymbol{C}+\boldsymbol{K}+\boldsymbol{S}_{K}+\boldsymbol{D}_{G} & \boldsymbol{G}+\boldsymbol{S}_{G} \\
\boldsymbol{D}+S_{D} & \boldsymbol{L}
\end{array}\right]\left[\begin{array}{c}
\boldsymbol{U} \\
\boldsymbol{P}
\end{array}\right]=\left[\begin{array}{c}
\boldsymbol{F}+\boldsymbol{S}_{F} \\
\boldsymbol{S}_{Q}+\boldsymbol{S}_{\Pi}
\end{array}\right]
$$

The different matrices in Eq. (47) represent the discrete form of the terms in Eqs. (44) and (45). Each one of them can be built by finite element assembly of elemental contributions. Using $a$ and $b$ to denote node indices and $i, j, k$ to denote coordinate directions, the block matrices in Eq. (47) that correspond to standard Galerkin terms can be defined as
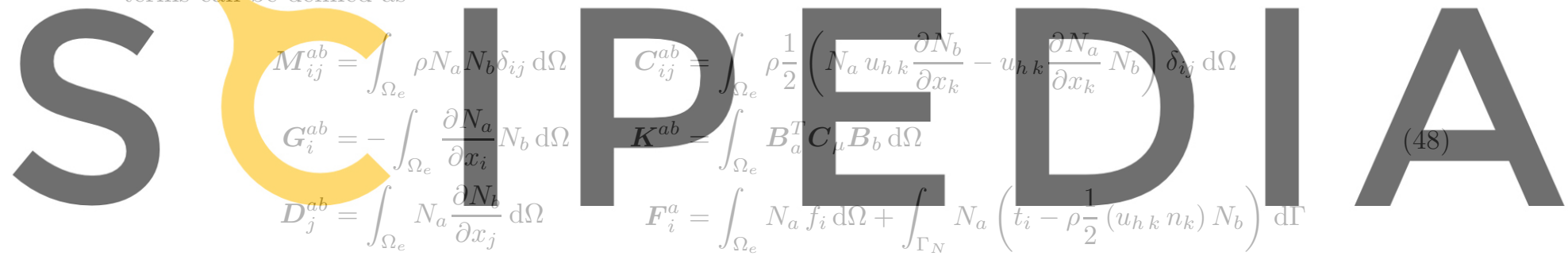

Register for free at https/swww.scipedia.com to download the version without the watermark corresponding to the contribution from node $a$, and the constitutive matrix $C_{\mu}$. For $3 D$ problems, these are defined by

$$
\boldsymbol{B}_{a}^{T}=\left[\begin{array}{cccccc}
\frac{\partial N_{a}}{\partial x} & 0 & 0 & \frac{\partial N_{a}}{\partial y} & 0 & \frac{\partial N_{a}}{\partial z} \\
0 & \frac{\partial N_{a}}{\partial y} & 0 & \frac{\partial N_{a}}{\partial x} & \frac{\partial N_{a}}{\partial z} & 0 \\
0 & 0 & \frac{\partial N_{a}}{\partial z} & 0 & \frac{\partial N_{a}}{\partial y} & \frac{\partial N_{a}}{\partial x}
\end{array}\right] \quad \boldsymbol{C}_{\mu}=\left[\begin{array}{cccccc}
4 \mu / 3 & -2 \mu / 3 & -2 \mu / 3 & 0 & 0 & 0 \\
-2 \mu / 3 & 4 \mu / 3 & -2 \mu / 3 & 0 & 0 & 0 \\
-2 \mu / 3 & -2 \mu / 3 & 4 \mu / 3 & 0 & 0 & 0 \\
0 & 0 & 0 & \mu & 0 & 0 \\
0 & 0 & 0 & 0 & \mu & 0 \\
0 & 0 & 0 & 0 & 0 & \mu
\end{array}\right]
$$

Similarly, the stabilization terms in Eq. (44) and Eq. (45) give rise to the following block matrices

$$
\begin{array}{rlrl}
\boldsymbol{M}_{K}^{a b}{ }_{i j}^{a b} & =\int_{\Omega_{e}} \rho \beta_{i} \frac{h_{u}}{2\|\boldsymbol{u}\|}\left(u_{h k} \frac{\partial N_{a}}{\partial x_{k}}\right) N_{b} \delta_{i j} \mathrm{~d} \Omega & \boldsymbol{S}_{D i}^{a b} & =\int_{\Omega_{e}} \tau_{c} N_{a} \frac{\partial N_{b}}{\partial x_{i}} \mathrm{~d} \Omega \\
\boldsymbol{M}_{D_{i}^{a b}} & =\int_{\Omega_{e}} \rho \tau_{c} \frac{\partial N_{a}}{\partial x_{i}} N_{b} \mathrm{~d} \Omega & \boldsymbol{L}^{a b} & =\int_{\Omega_{e}} \tau_{c}\left(\frac{\partial N_{a}}{\partial x_{k}}\right)^{T} \frac{\partial N_{b}}{\partial x_{k}} \mathrm{~d} \Omega \\
\boldsymbol{S}_{K}^{a b} & =\int_{\Omega_{e}} \rho \beta_{i} \frac{h_{u}}{2\|\boldsymbol{u}\|}\left(u_{h k} \frac{\partial N_{a}}{\partial x_{k}}\right)\left(u_{h l} \frac{\partial N_{b}}{\partial x_{l}}\right) \mathrm{d} \Omega & \boldsymbol{S}_{F}^{a} & =\int_{\Omega_{e}} \beta_{i} \frac{h_{u}}{2\|\boldsymbol{u}\|}\left(u_{h k} \frac{\partial N_{a}}{\partial x_{k}}\right) f_{i} \mathrm{~d} \Omega \\
\boldsymbol{S}_{G_{i}^{a b}} & =\int_{\Omega_{e}} \beta_{i} \frac{h_{u}}{2\|\boldsymbol{u}\|}\left(u_{h k} \frac{\partial N_{a}}{\partial x_{k}}\right) \frac{\partial N_{b}}{\partial x_{i}} \mathrm{~d} \Omega & \boldsymbol{S}_{Q}^{a} & =\int_{\Omega_{e}} \tau_{c} \frac{\partial N_{a}}{\partial x_{i}} f_{i} \mathrm{~d} \Omega \\
\boldsymbol{D}_{G_{i j}^{a b}} & =\int_{\Omega_{e}}\left(1-\beta_{i}\right)\left(\frac{\partial N_{a}}{\partial x_{k}}\right) D_{i j}^{g}\left(\frac{\partial N_{b}}{\partial x_{k}}\right) \mathrm{d} \Omega & \boldsymbol{S}_{\Pi}^{a}=-\int_{\Omega_{e}} \tau_{c} \frac{\partial N_{a}}{\partial x_{i}} \pi_{i} \mathrm{~d} \Omega
\end{array}
$$


Eq. (47) can be written using a compact notation as

$$
\tilde{\boldsymbol{M}}\left[\begin{array}{c}
\dot{U} \\
\mathbf{0}
\end{array}\right]+\tilde{C}\left[\begin{array}{l}
\boldsymbol{U} \\
\boldsymbol{P}
\end{array}\right]=\tilde{\boldsymbol{F}}
$$

If projections are used in the stabilization of the incompressibility equation, an additional system has to be solved to determine the nodal values of the projection variables $\boldsymbol{\pi}$. The equations for the projection can be obtained from the discrete version of Eq. (42), resulting in

$$
M_{\Pi} \Pi=R_{\Pi}
$$

where $\boldsymbol{\Pi}$ is the array of nodal values for the projection variables and

$$
\begin{aligned}
\boldsymbol{M}_{\Pi i j}^{a b} & =\int_{\Omega_{e}} N_{a} N_{b} \delta_{i j} \mathrm{~d} \Omega \\
\boldsymbol{R}_{\Pi}^{a} & =\int_{\Omega_{e}} N_{a} \hat{r}_{i}^{m} \mathrm{~d} \Omega
\end{aligned}
$$

Note that the assembly of elemental contributions given by Eq. (53) results in a dense matrix. In practice, the system matrix $\boldsymbol{M}_{\Pi}$ in Eq. (52) can be approximated by a diagonal mass matrix for efficiency.

\subsection{Time integration and linearization}

The problem described by Eqs. (44) and (45) involves the acceleration of the fluid. We need to introduce a time discretization to write the accelerations in terms the velocities. For this we have used the Bossak time scheme (see [28]). The Bossak method is a generalization of the Newmark method based on introducing a relaxation factor $\alpha_{B}$ for the acceleration of the system

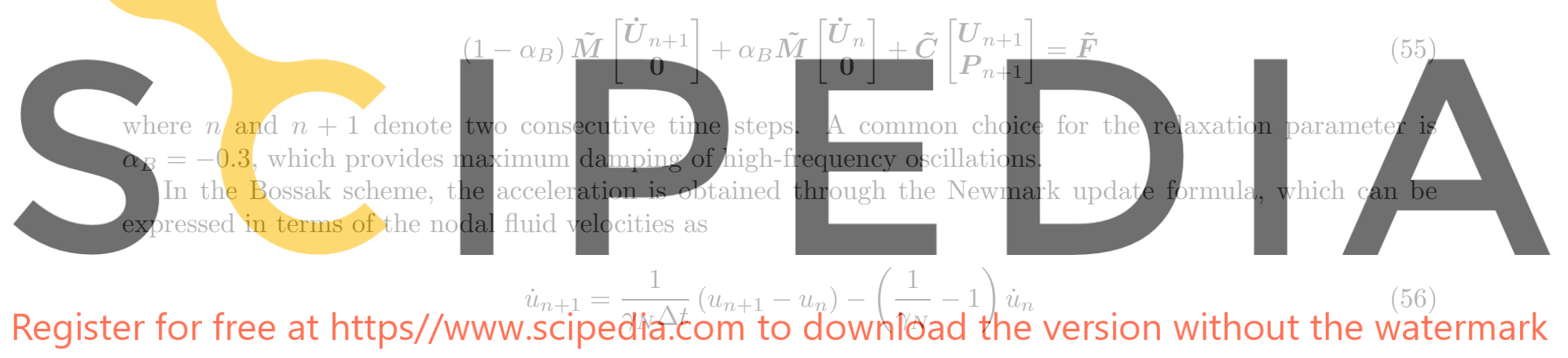

where the Newmark parameter $\gamma_{N}$ is given by

$$
\gamma_{N}=\frac{1}{2}-\alpha_{B}
$$

Introducing Eq. (56) in Eq. (55) we obtain the following time-discrete problem, where the velocities and pressures at the new time step are the only unknowns

$$
\left(\frac{1-\alpha_{B}}{\gamma_{N} \Delta t} \tilde{\boldsymbol{M}}+\tilde{\boldsymbol{C}}\right)\left[\begin{array}{l}
\boldsymbol{U}_{n+1} \\
\boldsymbol{P}_{n+1}
\end{array}\right]=\tilde{\boldsymbol{F}}-\frac{1-\alpha_{B}}{\gamma_{N} \Delta t} \tilde{\boldsymbol{M}}\left[\begin{array}{c}
\boldsymbol{U}_{n} \\
\mathbf{0}
\end{array}\right]+\left\{\left(1-\alpha_{B}\right)\left(\frac{1}{\gamma_{N}}-1\right)+\alpha_{B}\right\} \tilde{\boldsymbol{M}}\left[\begin{array}{c}
\dot{\boldsymbol{U}}_{n} \\
\mathbf{0}
\end{array}\right]
$$

Eq. (58) describes a system of non-linear equations in terms of the nodal values of velocity and pressure at time $t=(n+1) \Delta t$. The non-linearity arises from the fact that both the system matrix and the right-hand side term contain terms which depend on the current values of the variables, namely all terms involving the convective term $u_{k} \partial u_{i} / \partial x_{k}$, stabilization terms due to the dependence of the different stabilization coefficients on the local velocity $u_{i}$ and the gradient diffusion term, which involves both the momentum residual $r_{i}^{m}$ and the velocity gradients $\nabla u_{i}$. To obtain a linearized problem, we rewrite Eq. (58) in residual form. Defining the value of the unknowns at time step $n+1$ after $i$ non-linear iterations as $\boldsymbol{U}_{n+1}^{i}$, the linearized problem can be written as

$$
\begin{aligned}
\boldsymbol{R}_{n+1}^{i}\left(\boldsymbol{U}_{n+1}^{i}, \boldsymbol{P}_{n+1}^{i}\right)= & \tilde{\boldsymbol{F}}^{i}-\frac{1-\alpha_{B}}{\gamma_{N} \Delta t} \tilde{\boldsymbol{M}}^{i}\left[\begin{array}{c}
\boldsymbol{U}_{n} \\
\mathbf{0}
\end{array}\right] \\
& +\left\{\left(1-\alpha_{B}\right)\left(\frac{1}{\gamma_{N}}-1\right)+\alpha_{B}\right\} \tilde{\boldsymbol{M}}^{i}\left[\begin{array}{c}
\dot{\boldsymbol{U}}_{n} \\
\mathbf{0}
\end{array}\right]-\left(\frac{1-\alpha_{B}}{\gamma_{N} \Delta t} \tilde{\boldsymbol{M}}^{i}+\tilde{\boldsymbol{C}}^{i}\right)\left[\begin{array}{l}
\boldsymbol{U}_{n+1}^{i} \\
\boldsymbol{P}_{n+1}^{i}
\end{array}\right]
\end{aligned}
$$


where the index $i$ is used to denote that a quantity was computed using the values of velocity and pressure of iteration $i$.

Defining the increments between two successive iterations as $\boldsymbol{\delta} \boldsymbol{U}^{\boldsymbol{i}}=\boldsymbol{U}_{n+1}^{i+1}-\boldsymbol{U}_{n+1}^{i}$ and $\boldsymbol{\delta} \boldsymbol{P}^{\boldsymbol{i}}=\boldsymbol{P}_{n+1}^{i+1}-\boldsymbol{P}_{n+1}^{i}$, we can obtain a fixed point scheme to solve Eq. (59) by imposing that $\boldsymbol{R}_{n+1}^{i}\left(\boldsymbol{U}_{n+1}^{i}+\boldsymbol{\delta} \boldsymbol{U}^{\boldsymbol{i}}, \boldsymbol{P}_{n+1}^{i}+\boldsymbol{\delta} \boldsymbol{P}^{\boldsymbol{i}}\right)=0$. Assuming that all matrices are evaluated at step $i$, this yields

$$
-\left(\frac{1-\alpha_{B}}{\gamma_{N} \Delta t} \tilde{\boldsymbol{M}}+\tilde{\boldsymbol{C}}\right)\left[\begin{array}{l}
\boldsymbol{\delta} \boldsymbol{U}^{\boldsymbol{i}} \\
\boldsymbol{\delta} \boldsymbol{P}^{\boldsymbol{i}}
\end{array}\right]=\boldsymbol{R}_{n+1}^{i}\left(\boldsymbol{U}_{n+1}^{i}, \boldsymbol{P}_{n+1}^{i}\right)
$$

The problem given by Eq. (60) is solved iteratively until the increments $\boldsymbol{\delta} \boldsymbol{U}^{\boldsymbol{i}}$ and $\boldsymbol{\delta} \boldsymbol{P}^{\boldsymbol{i}}$ or the residual vector $\boldsymbol{R}_{n+1}^{i}\left(\boldsymbol{U}_{n+1}^{i}, \boldsymbol{P}_{n+1}^{i}\right)$ are smaller than a predefined tolerance.

\subsection{Summary of the FIC-FEM solution scheme}

Starting from the weak form described by Eq. (44) and Eq. (45), we have introduced a finite element discretization in space and a time discretization based on the Bossak method. Additionally, a Picard linearization has been used to obtain a linear system of equations to be solved iteratively, given by Eq. (60). To summarize, the complete FIC-FEM solution procedure is presented in compact form in Box 1.

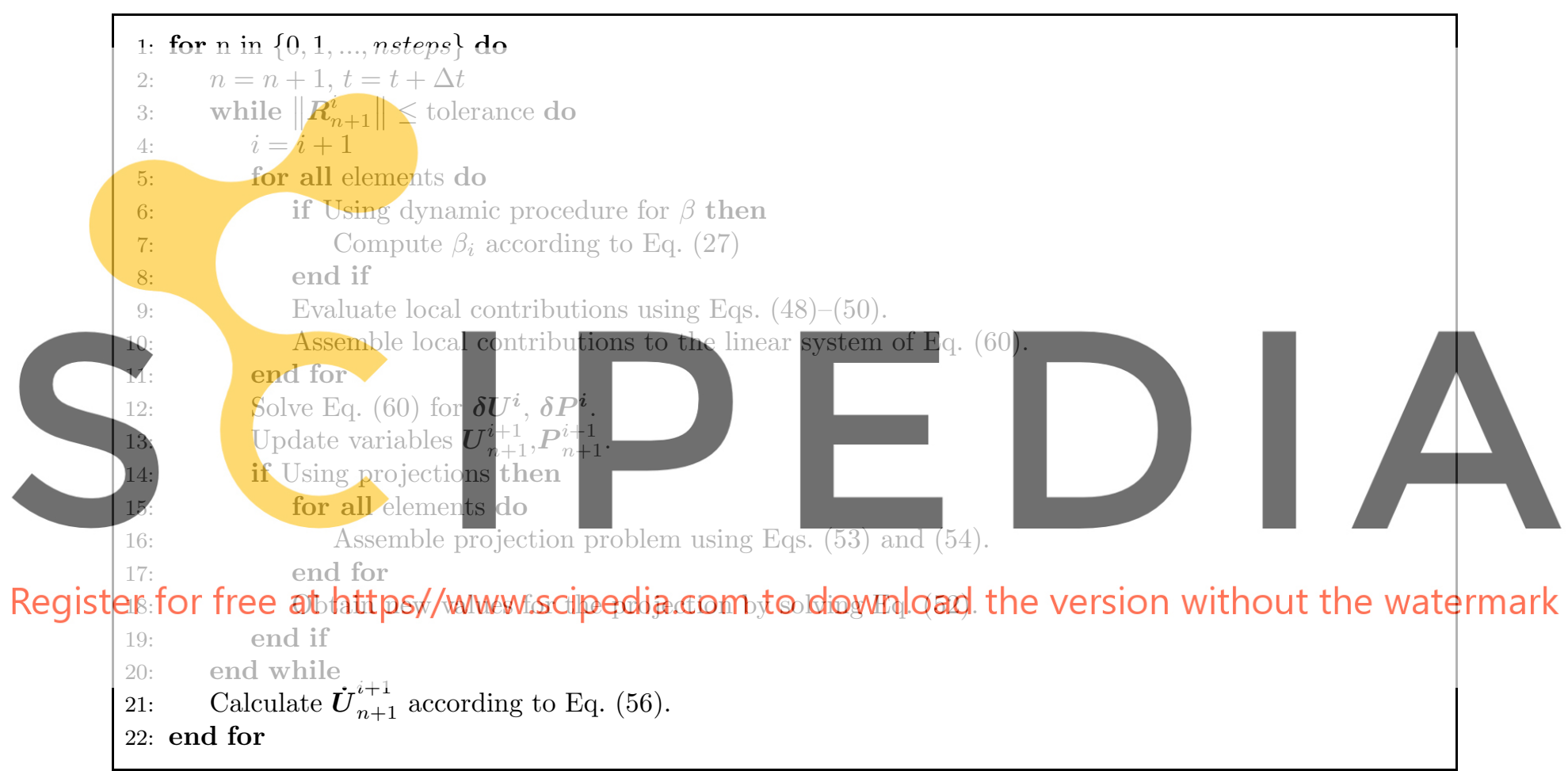

Box 1: FIC-FEM incompressible flow solver.

This formulation has been implemented within the Kratos Multiphysics code [29], a software framework for the development of finite element solvers.

\section{Example 1. Turbulent channel flow}

The flow in a plane turbulent channel is a classic turbulence benchmark and represents a challenging problem for LES formulations, due to the dependence of the vortex size to the distance to the wall [30]. It has been studied for a wide range of Reynolds numbers, but we direct our attention to the moderate value of $\operatorname{Re}_{\tau}=395$. There is an extensive bibliography regarding this case, with a comprehensive set of statistical data obtained from direct numerical simulations by Moser and co-authors [31]. In particular, this problem has been simulated using a variety of stabilized formulations, both using classical finite elements $[4,5,6,16]$ and isogeometric elements $[14,13]$.

This Reynolds number is very convenient because it allows us to use a mesh with enough resolution close to the wall while keeping the computational cost under control. At higher Reynolds numbers, the number of elements required to have a mesh fine enough for LES-type models close to the wall increases prohibitively and some type of wall model is usually preferable (see for example [32] or [33]). 
The plane turbulent channel problem simulates a flow driven by a fixed pressure gradient between two parallel infinite walls. Defining the distance between the two walls as $2 \delta$, the problem is formulated in terms of the wall friction $\tau_{w}$ and the friction velocity $u_{\tau}$

$$
\tau_{w}=-\delta \frac{\mathrm{d} P}{\mathrm{~d} x}=\rho u_{\tau}^{2} \quad \text { or } \quad u_{\tau}=\left(-\frac{\delta}{\rho} \frac{\mathrm{d} P}{\mathrm{~d} x}\right)^{\frac{1}{2}}
$$

where $\mathrm{d} P / \mathrm{d} x$ is the imposed pressure gradient. Using the definitions of Eq. (61), the turbulent channel problem can be characterized by the friction Reynolds number $\operatorname{Re}_{\tau}$, defined as

$$
\operatorname{Re}_{\tau}=\frac{u_{\tau} \delta}{\nu}
$$

which is set to $\operatorname{Re}_{\tau}=395$ for the present simulation. The results are presented in terms of the dimensionless distance to the wall $y+=u_{\tau} y / \nu$.

To perform the simulation at the desired Reynolds number, we have selected the following parameters:

$$
\begin{aligned}
\rho & =1 \mathrm{Kg} / \mathrm{m}^{3} & \nu & =1.472 \times 10^{-4} \mathrm{~m}^{2} / \mathrm{s} \\
\delta & =1 \mathrm{~m} & \frac{\mathrm{d} P}{\mathrm{~d} x} & =-3.372040 \times 10^{-3} \mathrm{~N} / \mathrm{m}^{2}
\end{aligned}
$$

\subsection{Problem definition}

We model a domain defined by $[0,2 \pi] \times[-\delta, \delta] \times[0,2 \pi / 3]$ in the stream-wise, wall-normal and cross-stream directions respectively, using the same domain as in [14]. Zero velocity Dirichlet conditions are assigned on the wall sides and periodic boundary conditions are used in the remaining directions.

The problem has been modeled using linear hexahedral or tetrahedral elements. Mesh nodes are placed regularly along the stream-wise and cross-stream directions, while a weighting function is used in the wallnormal direction to move the nodes closer to the wall. The location $y^{i}$ of the i-th node in the wall-normal direction is chosen as

$$
y^{i}=\delta \frac{\tanh \left(w\left(\frac{2 i}{n_{e l}}-1\right)\right)}{\tanh (w)} \quad i \in\left[0, n_{e l}\right]
$$

with $w=2.432$ for $n_{e l}=64$ and $w=2.927$ for $n_{e l}=32$, chosen so that the first layer of internal nodes is at a dimensionless distance to the wall $y+=1$. For the tetrahedral meshes, the hexahedral space defined by successive nodes along each direction is split into six tetrahedra.

The time step for the simulation is chosen as $\Delta t=0.04 \mathrm{~s}$ which, according to the analysis in [34], is sufficient to reproduce the features of the flow. We use the expected average velocity profile as the initial condition, adding a random fluctuation in the wall-normal direction to destabilize the flow. Once an unsteady solution develops, the flow is left to evolve until it reaches a statistically homogeneous regime. Statistical results are collected by sampling the quantities of interest at the integration points of each element, averaging over time and over planes parallel to the walls.

We have performed multiple simulations using these settings to study the behavior of different variants of the FIC-FEM formulation presented in the preceding pages. Note that all turbulent channel flow cases were run using the projections for the mass stabilization in Eq. (43).

\subsection{Fixed combination parameter}

The first set of test cases use the FIC formulation with a fixed combination parameter. We introduce the notation $\langle x\rangle$ to describe the average of quantity $x$ (in time and over directions of statistical homogeneity) and $x^{\prime}:=x-\langle x\rangle$ to denote its fluctuation. The average stream-wise velocity $\langle u\rangle$, relative to the friction velocity $u_{\tau}$, is shown in Fig. 2(a) for tetrahedral meshes and in Fig. 2(c) for hexhahedra. Both figures also show the DNS results of Moser et al. [31] for the same Reynolds number, which we adopt as a reference solution.

In addition to studying the solution in terms of the average flow, we are also interested in quantifying the fluctuation in our solution, which should be properly captured if we want to represent turbulent flows accurately. We introduce the distribution of turbulence kinetic energy along the wall-normal direction as a second quantity of interest. Turbulence kinetic energy is defined in terms of the velocity variances as

$$
k=\frac{1}{2}\left(\left\langle u^{\prime} u^{\prime}\right\rangle+\left\langle v^{\prime} v^{\prime}\right\rangle+\left\langle w^{\prime} w^{\prime}\right\rangle\right)
$$

and is shown in Fig. 2(a) for the cases simulated using tetrahedral meshes and in Fig. 2(d) for hexahedra, again compared to the DNS reference solution. In addition, the individual velocity variance components in Eq. (65) are presented in the plots of Fig. 3. 


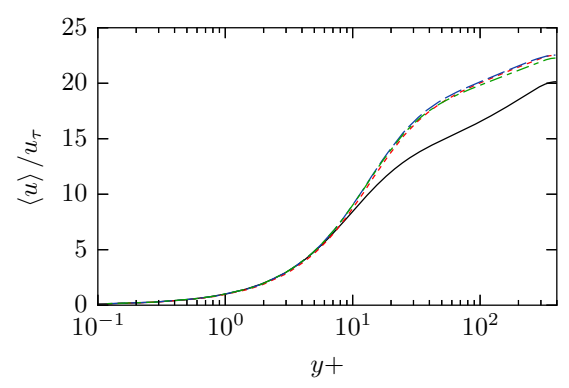

(a) Average stream-wise velocity (tetrahedra).

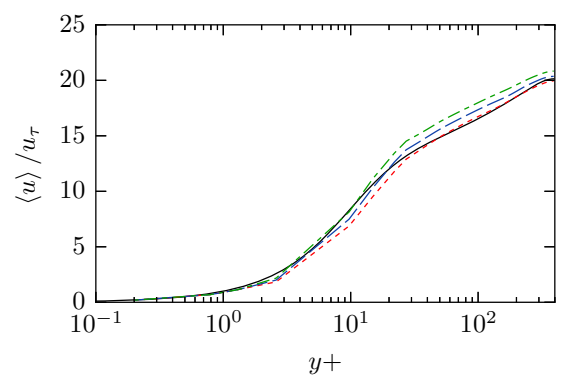

(c) Average stream-wise velocity (hexahedra).

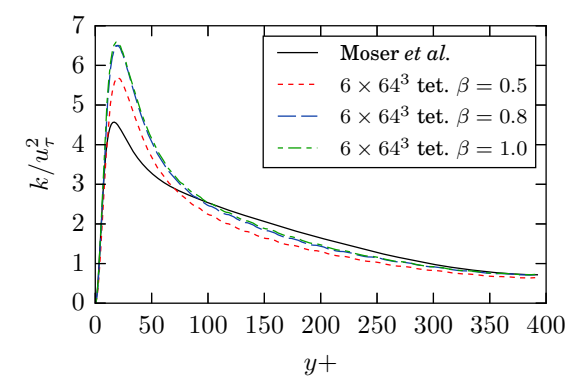

(b) Turbulence kinetic energy (tetrahedra).

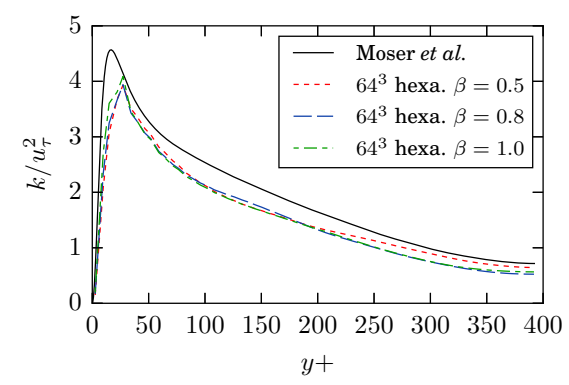

(d) Turbulence kinetic energy (hexahedra).

Figure 2: Channel flow - average stream-wise velocity and turbulence kinetic energy profiles obtained using hexahedral or tetrahedral elements elements and a fixed $\beta$, compared to the DNS data of Moser et al. [31].

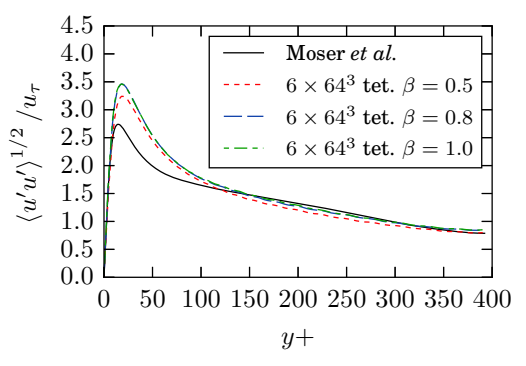

(a) Stream-wise fluctuation $\left\langle u^{\prime} u^{\prime}\right\rangle$.

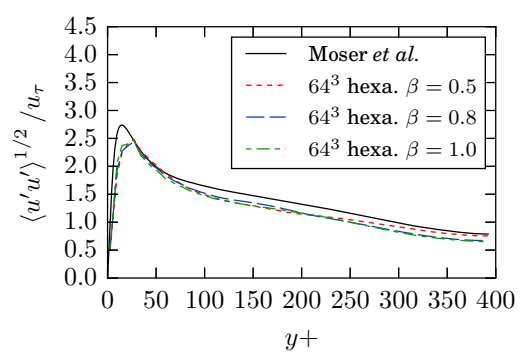

(d) Stream-wise fluctuation $\left\langle u^{\prime} u^{\prime}\right\rangle$.

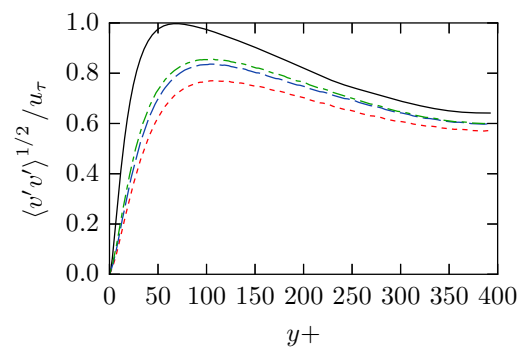

(b) Wall-normal fluctuation $\left\langle v^{\prime} v^{\prime}\right\rangle$.

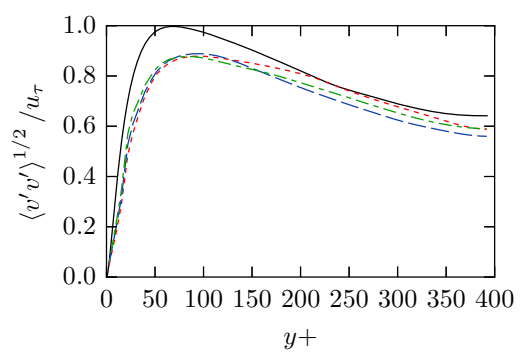

(e) Wall-normal fluctuation $\left\langle v^{\prime} v^{\prime}\right\rangle$.

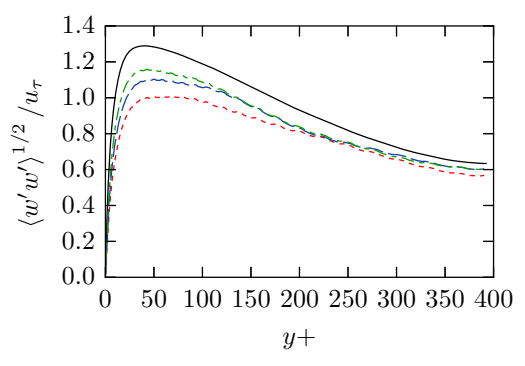

(c) Cross-stream fluctuation $\left\langle w^{\prime} w^{\prime}\right\rangle$.

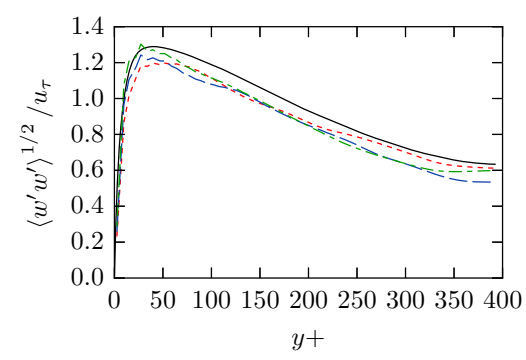

(f) Cross-stream fluctuation $\left\langle w^{\prime} w^{\prime}\right\rangle$.

Figure 3: Channel flow - Reynolds stresses obtained using hexahedral or tetrahedral elements using a fixed $\beta$, compared to Moser et al. [31]. 
Finally, we measure the dissipation of average stream-wise linear momentum in the wall-normal direction. We know from studying the RANS momentum equation that the total mean shear stress in the $x y$ plane can be written as (see for example [30] or [3])

$$
\left\langle\tau_{x y}\right\rangle=-\rho\left\langle u^{\prime} v^{\prime}\right\rangle+\mu \frac{\partial\langle u\rangle}{\partial y}=\tau_{w} \frac{y}{\delta}
$$

where the first term of the middle equality represents the Reynolds stresses in the $x y$ plane and the second the viscous stress due to the average velocity. This decomposition is shown in Fig. 4 for the simulation performed using hexahedral elements and $\beta=0.8$. The balance of stresses for the average solution implies that the total mean shear stress $\left\langle\tau_{x y}\right\rangle$ is equal to $\rho u_{\tau}^{2}$ (in module) at the walls and varies linearly on the wall-normal direction (see for example [30]). The fact that the addition of the two terms, as measured in the simulation, is close to the expected straight line allows us to verify that the solution has reached statistical equilibrium.

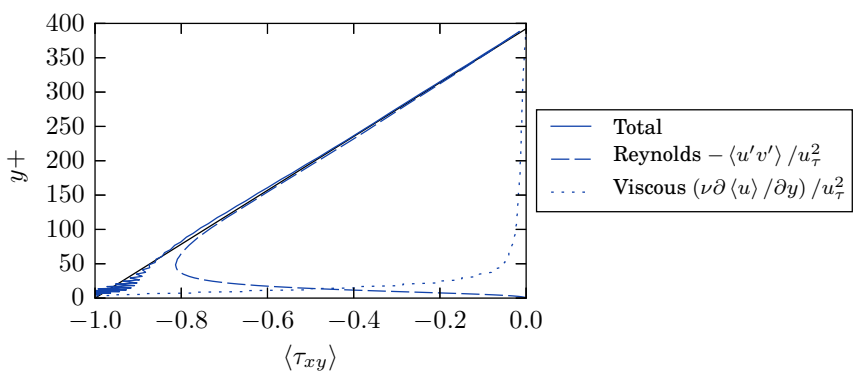

Figure 4: Channel flow - average $\left\langle\tau_{x y}\right\rangle$ stress profile obtained using a mesh with $64^{3}$ hexahedra and fixing $\beta=0.8$.

We observe that the results obtained with linear hexahedra are closer to the reference values than those obtained with linear tetrahedra in all cases. This can also be seen in Fig. 5, which shows both the tetrahedral and hexhahedral solutions for $\beta=0.8$. This is to be expected, as hexahedra use trilinear shape functions, which define a richer interpolation than the linear functions used in tetrahedra.

Figs. 2 and 3 give us an idea of the dependence of the solution on the free parameter of the formulation, $\beta$, which is set a priori. We observe that there is not a large amount of variation between the different cases, although the $\beta=0.5$ case tends to display a lower level of velocity fluctuations in all directions and, conversely, large values of $\beta$ produce more fluctuation. This suggests that small values of $\beta$, which give more weight to the gradient diffusion term, result in a solution that is more diffusive overall.

\subsection{Variable combination parameter}

The next set of tests has been performed using Eq. (27) to assign a value to the combination parameter $\beta_{i}$, while keeping the remaining simulation settings as in the fixed beta case. In a first round, we set the minimum value of the coefficient to $\beta_{m}=0.8$ and simulate the problem using both hexahedral and tetrahedral meshes. The averaged results of the simulation are presented in Fig. 5, where they are compared to those obtained for a fixed coefficient in the previous section.

We can see in the figures that the average velocity profiles are generally lower to those obtained in the fixed $\beta$ cases and in better agreement with DNS data. Conversely, velocity fluctuations measured in the stream-wise direction are somewhat lower than in the fixed $\beta$ simulations, resulting in a turbulence kinetic energy that is closer to that measured in the reference DNS simulations.

As in the previous case, we want to measure the impact on the results of the choice of the limit value $\beta_{m}$ for the combination coefficient. To test its influence, we have run several simulations for tetrahedra (Fig. 6) and hexahedra (Fig. 7), changing the value of $\beta_{m}$. Results in good agreement with the reference DNS data are obtained for $\beta_{i} \geq 0.8$ and $\beta_{i} \geq 0.9$. In general, results results show limited sensitivity to the choice of parameter, at least for large values of the $\beta$ parameter.

A different question regarding the behavior of the method is the sensitivity of the solution to the choice of mesh size. In addition to the presented simulations, we have repeated the case $\beta_{i} \geq 0.8$ using different mesh sizes, namely two coarser meshes composed of $32^{3}$ hexahedra and $6 \times 32^{3}$ tetrahedra as well as one finer case with a $128^{3}$ hexahedra mesh. The results for these simulations are presented in Fig. 8 for tetrahedra and Fig. 9 for hexahedra, compared to the previous results for the same case. Again, we notice a clear difference in the behaviour of tetrahedra and hexahedra. In particular, it seems that the coarser tetrahedral mesh is insufficient to reproduce the features of the problem, resulting in a significantly larger average velocity.

Finally, we present in Fig. 10 the energy spectrum corresponding to the solution of the case with a $32^{3}$ hexahedra mesh and a variable combination coefficient $\beta_{i} \geq 0.8$. This result has been calculated using the 


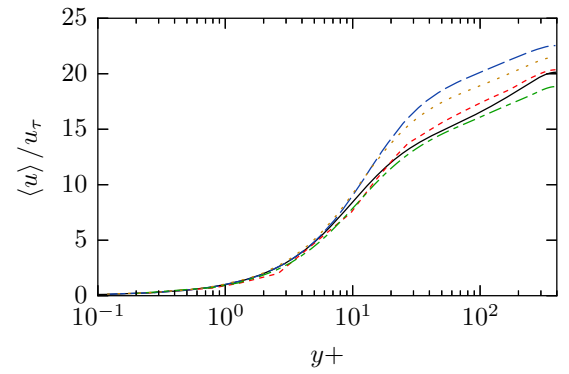

(a) Average stream-wise velocity

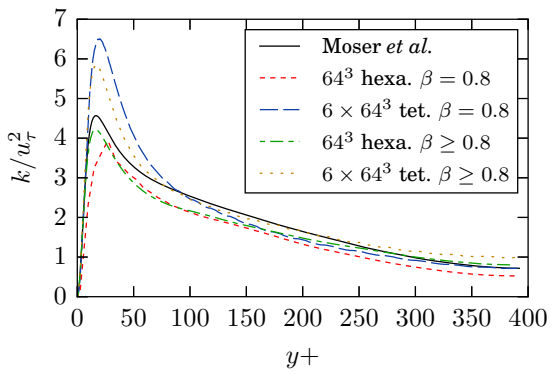

(b) Turbulence kinetic energy.

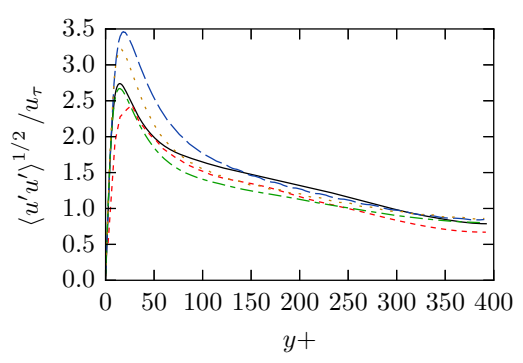

(c) Stream-wise fluctuation $\left\langle u^{\prime} u^{\prime}\right\rangle$.

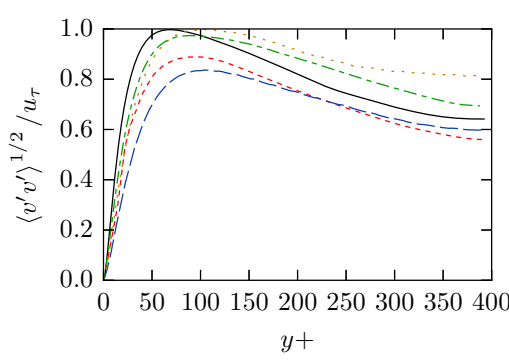

(d) Wall-normal fluctuation $\left\langle v^{\prime} v^{\prime}\right\rangle$.

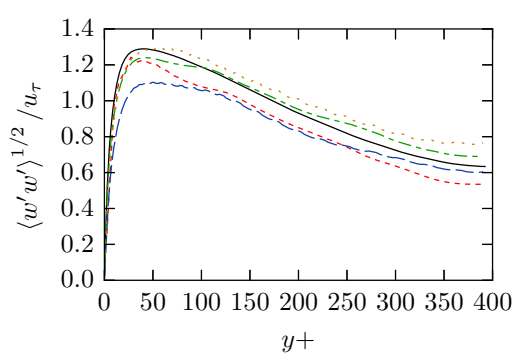

(e) Cross-stream fluctuation $\left\langle w^{\prime} w^{\prime}\right\rangle$.

Figure 5: Channel flow - velocity average and variances using a fixed or variable combination parameter.

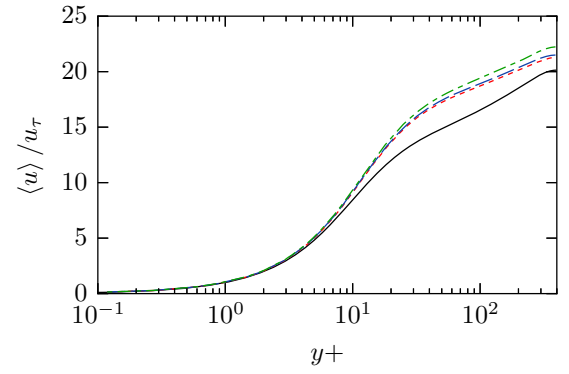

(a) Average stream-wise velocity

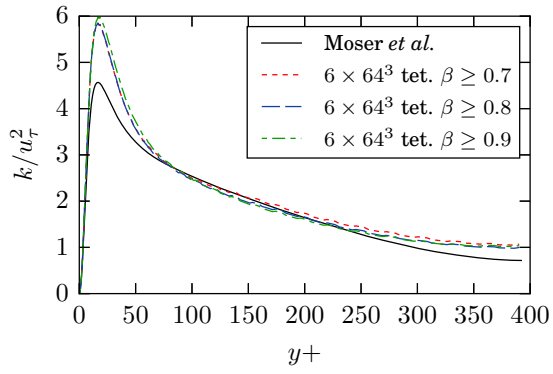

(b) Turbulence kinetic energy.

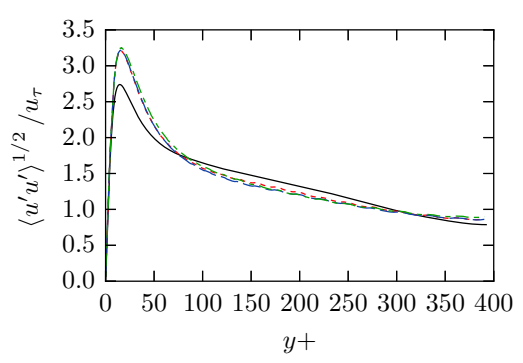

(c) Stream-wise fluctuation $\left\langle u^{\prime} u^{\prime}\right\rangle$.

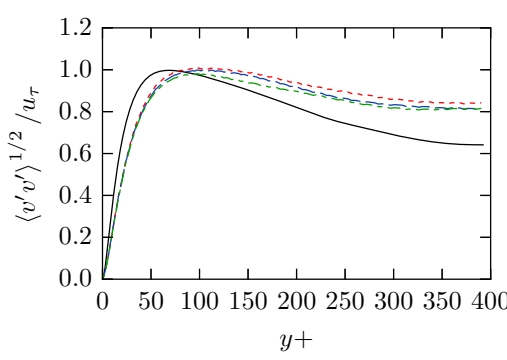

(d) Wall-normal fluctuation $\left\langle v^{\prime} v^{\prime}\right\rangle$.

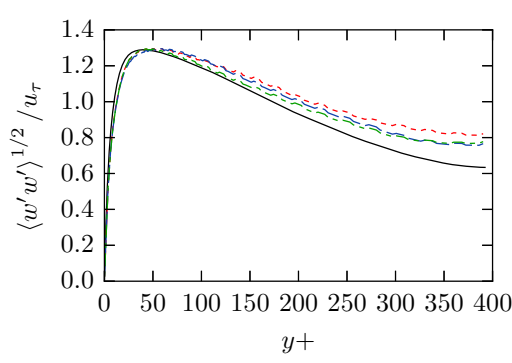

(e) Cross-stream fluctuation $\left\langle w^{\prime} w^{\prime}\right\rangle$.

Figure 6: Channel flow - velocity average and variances obtained using linear tetrahedra and different limits for a variable combination parameter. 


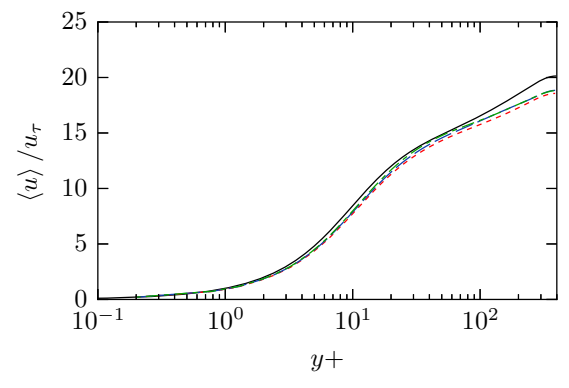

(a) Average stream-wise velocity

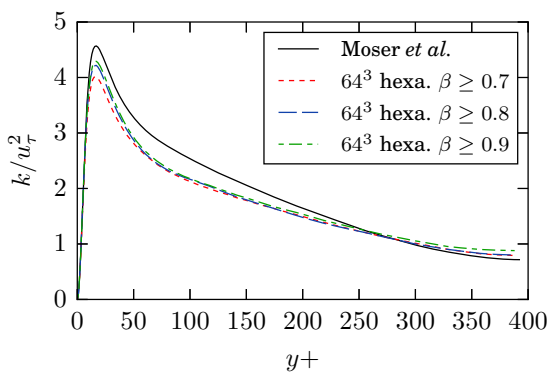

(b) Turbulence kinetic energy.

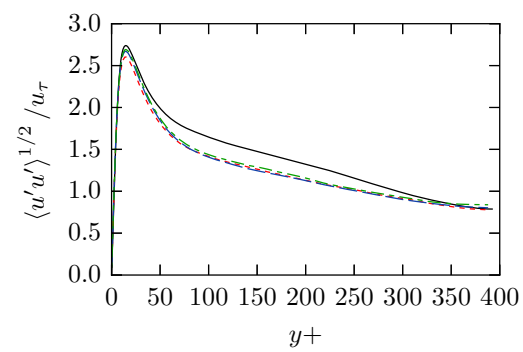

(c) Stream-wise fluctuation $\left\langle u^{\prime} u^{\prime}\right\rangle$.

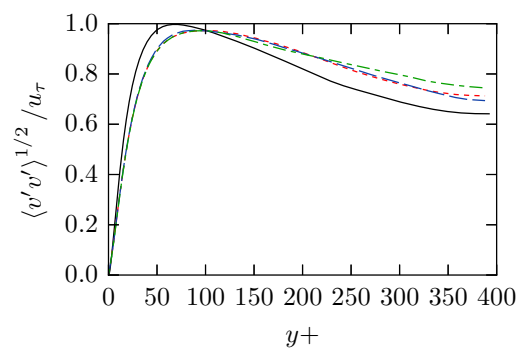

(d) Wall-normal fluctuation $\left\langle v^{\prime} v^{\prime}\right\rangle$.

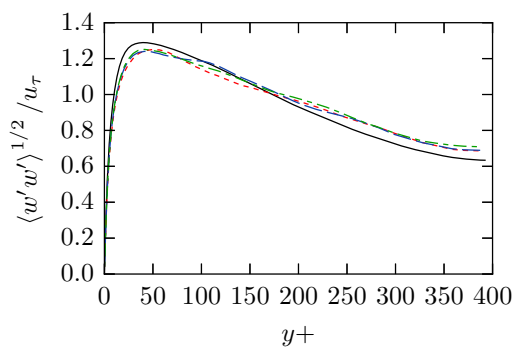

(e) Cross-stream fluctuation $\left\langle w^{\prime} w^{\prime}\right\rangle$.

Figure 7: Channel flow - velocity average and variances obtained using linear hexahedra and different limits for a variable combination parameter.

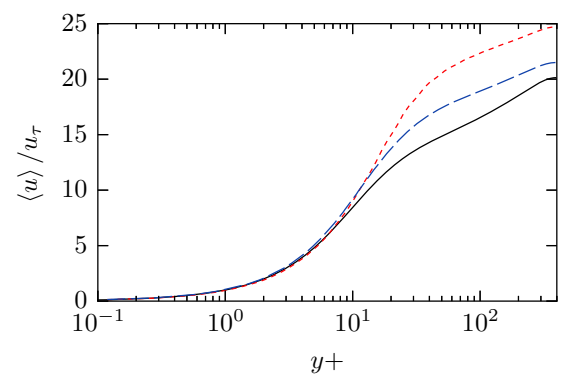

(a) Average stream-wise velocity

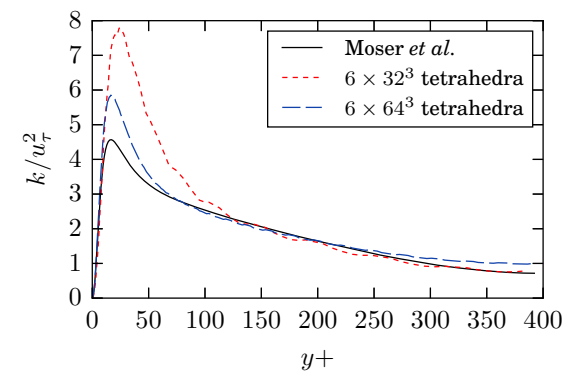

(b) Turbulence kinetic energy.

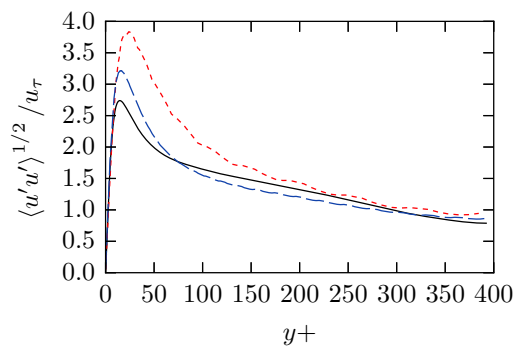

(c) Stream-wise fluctuation $\left\langle u^{\prime} u^{\prime}\right\rangle$.

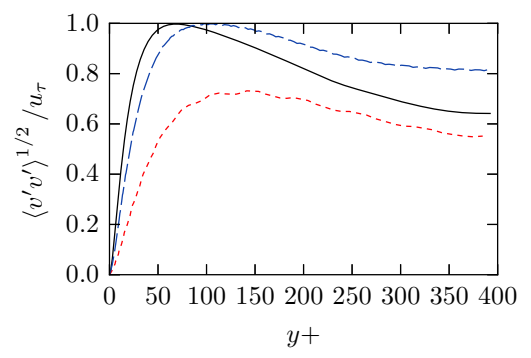

(d) Wall-normal fluctuation $\left\langle v^{\prime} v^{\prime}\right\rangle$.

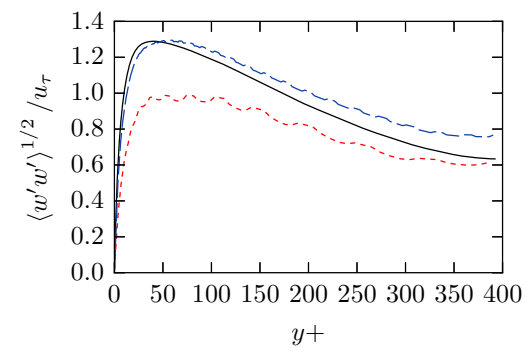

(e) Cross-stream fluctuation $\left\langle w^{\prime} w^{\prime}\right\rangle$.

Figure 8: Channel flow - velocity average and variances obtained using different grid sizes (all results with a variable $\left.\beta_{i} \geq 0.8\right)$. 


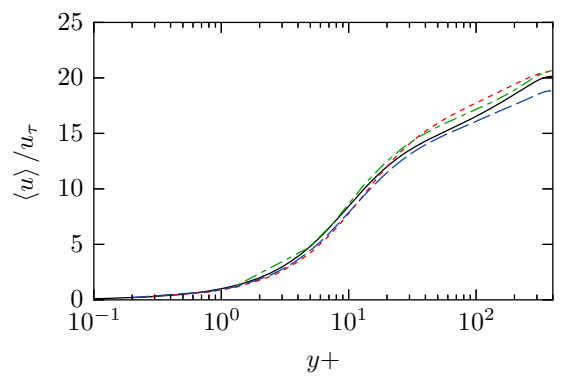

(a) Average stream-wise velocity

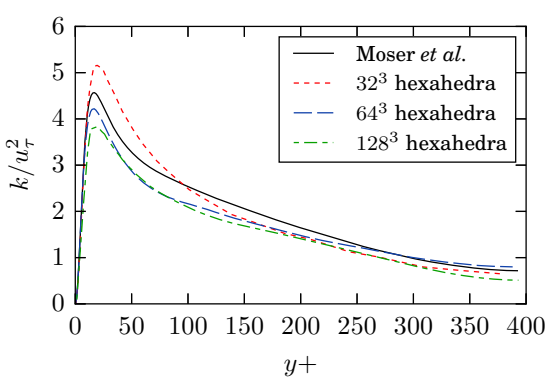

(b) Turbulence kinetic energy.

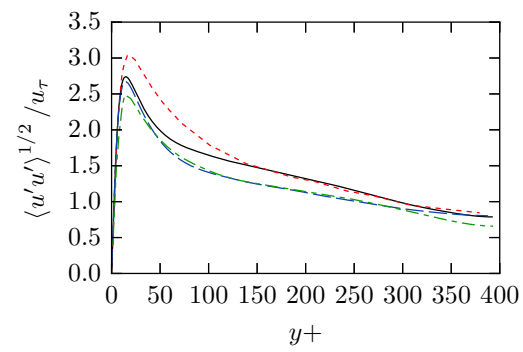

(c) Stream-wise fluctuation $\left\langle u^{\prime} u^{\prime}\right\rangle$.

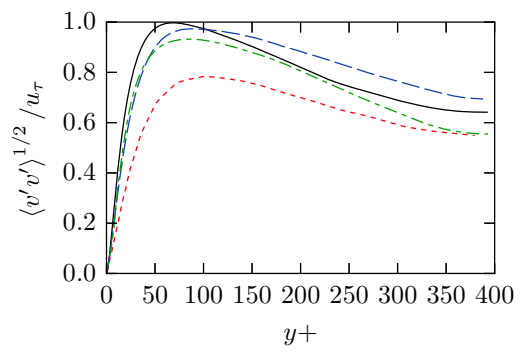

(d) Wall-normal fluctuation $\left\langle v^{\prime} v^{\prime}\right\rangle$.

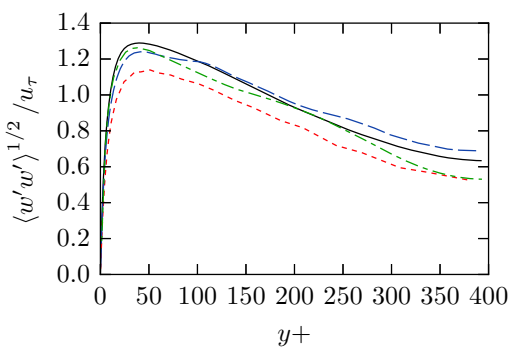

(e) Cross-stream fluctuation $\left\langle w^{\prime} w^{\prime}\right\rangle$.

Figure 9: Channel flow - velocity average and variances obtained using different grid sizes (all results with a variable $\beta_{i} \geq 0.8$ ).

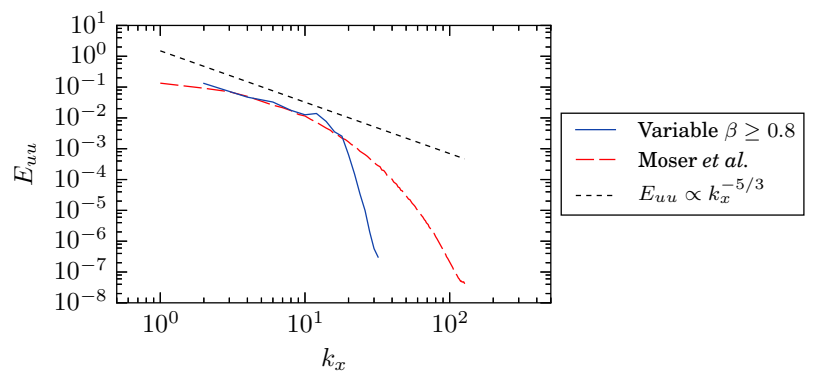

Figure 10: Channel flow - stream-wise energy spectrum obtained using a $32^{3}$ hexahedra mesh and a variable combination coefficient $\beta_{i} \geq 0.8$, compared to the DNS data of Moser et al. [31].

stream-wise velocity along the centerline of the channel and is found to be in agreement to the same measurement in the data of Moser et al. [31] up to the highest wavenumbers allowed by mesh resolution. In addition, the measured spectrum for the lower wavenumbers matches the theoretical slope of $k_{x}^{-5 / 3}$ expected in the inertial subrange, which has been plotted in the figure for reference.

\subsection{Summary of the results}

We summarize the main results for the analysis of the turbulent channel flow at $\operatorname{Re}_{\tau}=395$ test case. A first general observation is that hexahedra produce more accurate solutions than tetrahedra for a given mesh size. This was expected, since the interpolation space used for hexahedral meshes is richer, but our results allow us to quantify the difference in this case. A comparison of Figs. 8 and Fig. 9 suggests that $6 \times 64^{3}$ tetrahedra are required to obtain results comparable to $32^{3}$ hexahedra, which represents 48 times more elements in total.

Given that the formulation proposed here has a free parameter, the combination parameter $\beta_{i}$, we studied the sensitivity of the solution to its value. In the cases where $\beta_{i}$ is fixed throughout the simulation, the general trend is to obtain lower variances the smaller the coefficient, which is equivalent to giving more weight to the gradient diffusion term (see Fig. 3). This suggests that the gradient diffusion term introduces a significant amount of numerical dissipation, resulting in more homogeneous solutions. In comparison, the average velocity profile is less sensitive to the choice of parameter, as similar results are obtained in the different cases.

If the combination parameter $\beta_{i}$ is set on each element according to the local weighting function of Eq. (27), the obtained velocity profiles are generally lower than those obtained with a fixed parameter and measured variances show a better agreement with the DNS data. This is most noticeable for the $\left\langle u^{\prime} u^{\prime}\right\rangle$ correlation, which 


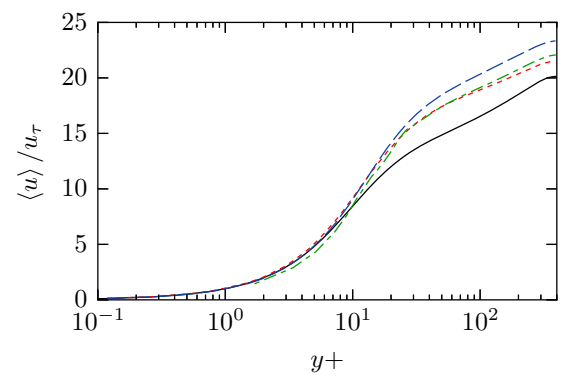

(a) Average stream-wise velocity

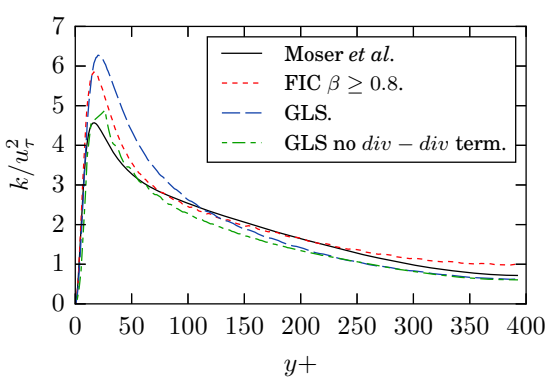

(b) Turbulence kinetic energy.

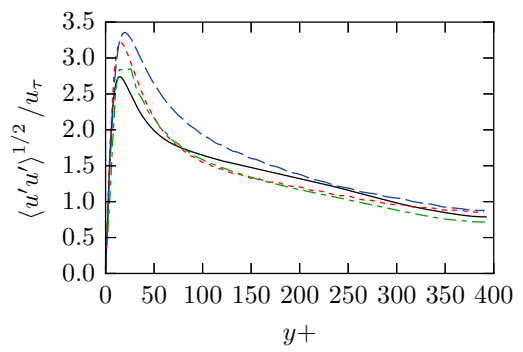

(c) Stream-wise fluctuation $\left\langle u^{\prime} u^{\prime}\right\rangle$.

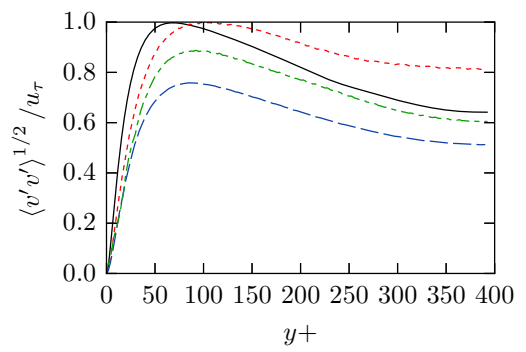

(d) Wall-normal fluctuation $\left\langle v^{\prime} v^{\prime}\right\rangle$.

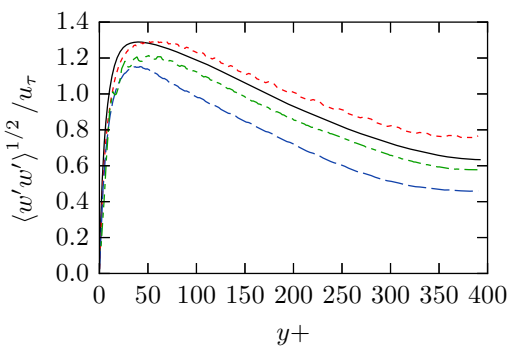

(e) Cross-stream fluctuation $\left\langle w^{\prime} w^{\prime}\right\rangle$.

Figure 11: Channel flow - velocity average and variances obtained using FIC-FEM with a variable combination coefficient $\beta_{i} \geq 0.8$ and the GLS method on a $6 \times 64^{3}$ tetrahedra mesh.

is the larger contribution to the total turbulence kinetic energy (see Fig. 5). Using this approach, there is still a parameter to be set externally, the minimum admissible value of $\beta$, but our results show little sensitivity to this parameter, as can be observed in Fig. 6 for tetrahedra and Fig. 7 for hexahedra.

As a final verification, we compare the presented FIC-FEM approach to the more standard Galerkin-Least squares (GLS) method. In practical terms, the main differences between the present formulation and GLS are the presence of the gradient diffusion term in the stabilized momentum equation Eq. (44) and the absence of a div-div term in the form $(\nabla \cdot \boldsymbol{w}) \tau_{2}(\nabla \cdot \boldsymbol{u})$, which in GLS is obtained from the mass balance equation. This div-div term is considered relevant in this case, since it has been shown to have a negative impact in the accuracy of the solution for this case (see for example [16]).

In order to quantify the relative importance of these two differences, we compare the results obtained using the present FIC-FEM approach with a variable $\beta_{i}$ to those produced by a GLS formulation on the same finite element meshes, both including and neglecting the div-div term. The results are presented in Fig. 11 for tetrahedral meshes and in Fig. 12 for hexahedral elements. Our results confirm the negative impact of the divdiv term in the solution and show that, in general, the FIC-FEM formulation results in slightly lower velocity averages than the GLS method without the div-div term. This is more noticeable in the hexahedral mesh while, for tetrahedra, the velocity profiles obtained by these two methods are very close.

\section{Example 2. Flow around a cylinder}

The flow over a circular cylinder is a classical problem in CFD simulations, which has been studied extensively, both experimentally and numerically (see for example the review in [35]). This problem exhibits a variable behavior depending on the Reynolds number, due to the different vortex shedding mechanisms that develop on the wake of the cylinder [36]. As a benchmark example for the FIC-FEM formulation, we study the case corresponding to a Reynolds number $\mathrm{Re}=3900$ based on the diameter of the cylinder and the inflow velocity, for which extensive data, both experimental [37] and numerical [38, 39], is available. For our tests, we adopt a domain and problem set up similar to that of [38] and compare our results to those presented in that reference.

We simulate the flow over a cylinder with diameter $D$ using a domain of $30 D \times 30 D$, centered on the cylinder, in the plane normal to the cylinder's axis, and $W=3.14 D$ in the span-wise direction. The simulation is run for 160 dimensionless time units (made dimensionless using the inflow velocity $U_{\infty}$ and the diameter $D$ ) with a dimensionless time step $\delta t=0.1$, which should provide sufficient resolution to capture the main vortex shedding frequency.

The domain for the problem is presented in Figure 13. Consider the axes $x, y$ and $z$, aligned in the streamwise, cross-stream and span-wise directions respectively, and let $u, v$ and $w$, be the components of the flow velocity in each of the three coordinate directions. We are interested in measuring the velocity history in 


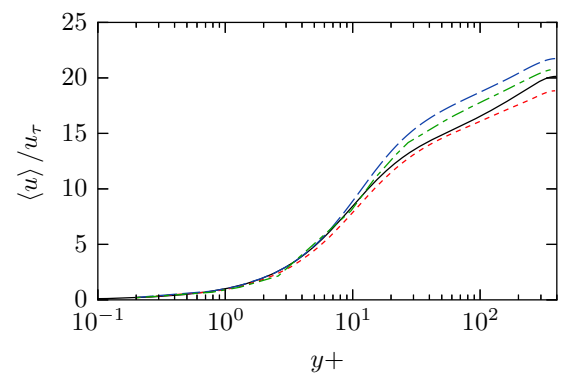

(a) Average stream-wise velocity

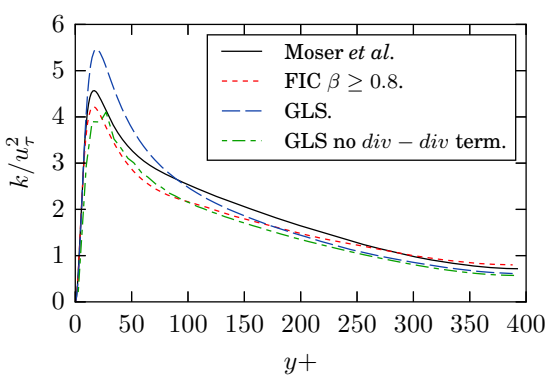

(b) Turbulence kinetic energy.

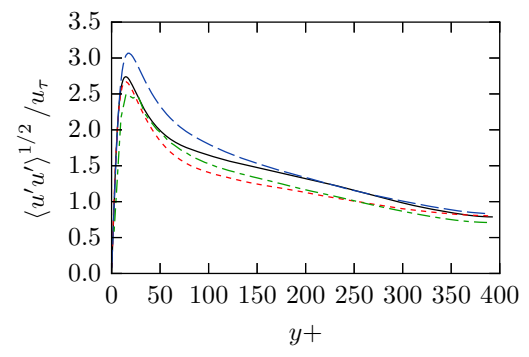

(c) Stream-wise fluctuation $\left\langle u^{\prime} u^{\prime}\right\rangle$.

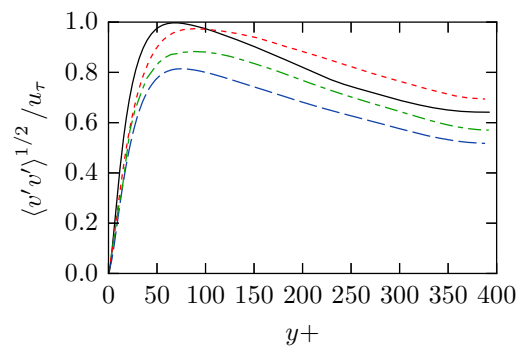

(d) Wall-normal fluctuation $\left\langle v^{\prime} v^{\prime}\right\rangle$.

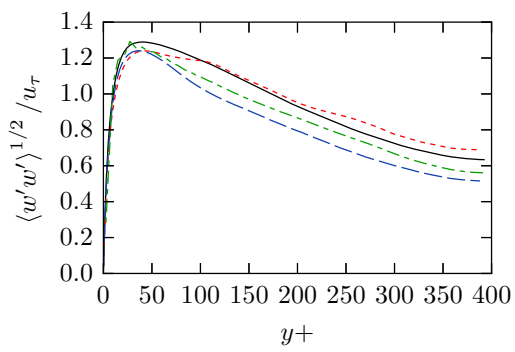

(e) Cross-stream fluctuation $\left\langle w^{\prime} w^{\prime}\right\rangle$.

Figure 12: Channel flow - velocity average and variances obtained using FIC-FEM with a variable combination coefficient $\beta_{i} \geq 0.8$ and the GLS method on a $64^{3}$ hexahedra mesh.

selected planes in the wake of the cylinder, pictured in Figure 13, chosen to coincide with those reported in the reference [38].

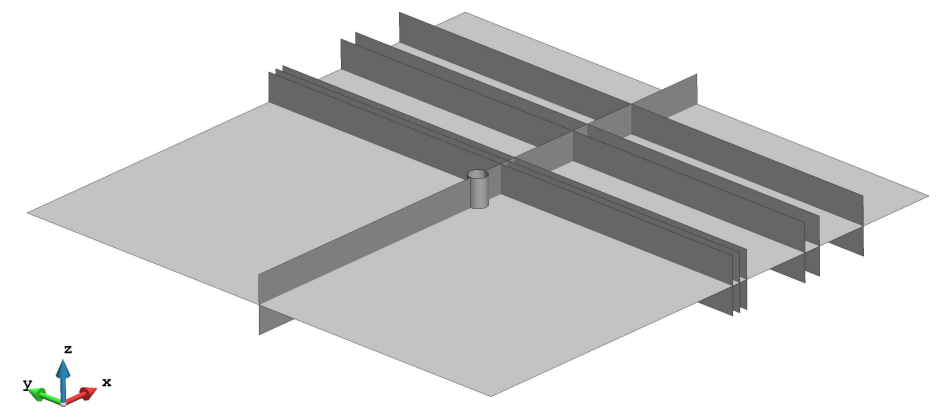

Figure 13: Flow around a cylinder - simulation domain and measurement planes.

The velocity is fixed to a constant $U_{\infty}=1 \mathrm{~m} / \mathrm{s}$ for the inlet and to zero on the cylinder surface. Periodic boundary conditions are used in the span-wise direction, while a no-penetration condition $v=0$ is set in the far sides along the cross-stream direction. Based on the results obtained for the channel flow, we decide to use the FIC variant with a variable combination parameter, limiting the combination parameter to a minimum value of $\beta_{m}=0.8$.

We have used four different meshes to simulate the problem, as reported in Table 1. The meshes are unstructured in all cases, with sizes ranging between $0.03 D$ near the cylinder to $0.5 D$ in the far regions, and higher element counts correspond to increasingly refined mesh sizes on the cylinder wake. It must be noted that, although hexahedral elements were shown to have a superior performance in the previous test case, we consider that tetrahedral are relevant in complex flows, due to the difficulty of meshing arbitrarily complex domains using hexahedra. All reported figures and results correspond to mesh T2 unless otherwise stated.

The different tetrahedral meshes have been used to ensure that mesh convergence is achieved, while the hexahedral case is intended as a comparison between the two element types, using a comparable number of mesh nodes. However, there is an important difference in the two meshes, due to limitations on the meshing procedure. While the tetrahedral meshes are completely unstructured, the hexahedral mesh is structured in the span-wise direction, since it was generated by translation of a quadrilateral mesh. This means that the hexahedral mesh has the same (smallest) mesh size along the span-wise dimension across the entire domain, resulting in elongated elements in the coarser regions.

We show the average velocity along several $y-z$ cross sections on the wake of the cylinder in Fig. 14, comparing 


\begin{tabular}{c|c|c|c} 
Mesh & Element type & Num. nodes $\left(\times 10^{6}\right)$ & Num. elements $\left(\times 10^{6}\right)$ \\
\hline T1 & Tetrahedra & 0.73 & 4.18 \\
T2 & Tetrahedra & 1.74 & 10.0 \\
T3 & Tetrahedra & 3.82 & 23.2 \\
H & Hexahedra & 1.8 & 1.75
\end{tabular}

Table 1: Flow around a cylinder - mesh definition.

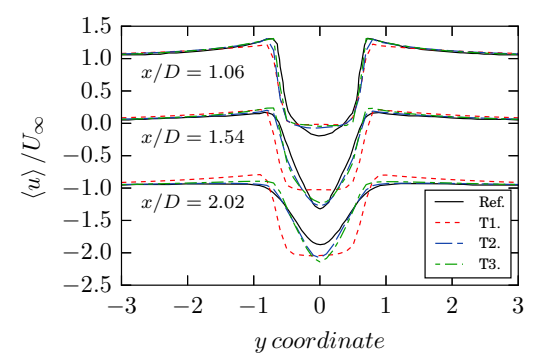

(a) Stream-wise velocity $\langle u\rangle$ (near wake).

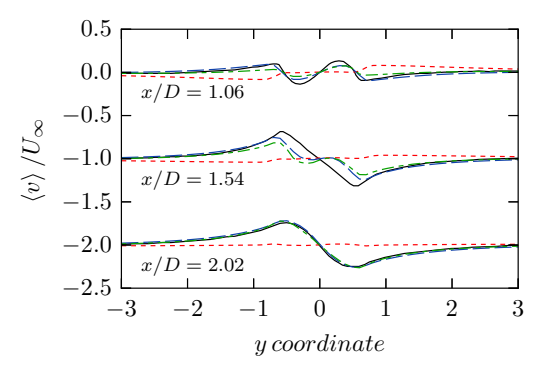

(b) Cross-stream velocity $\langle v\rangle$ (near wake).

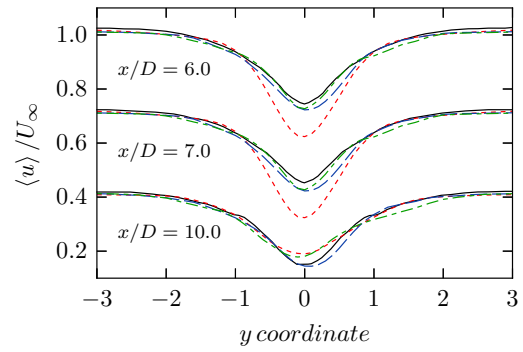

(c) Stream-wise velocity $\langle u\rangle$ (far wake).

Figure 14: Flow around a cylinder - average velocities in the wake for different tetrahedral meshes.

the results obtained for the different tetrahedral meshes to the results presented in [38], which we adopt as a reference solution. Since cases T2 and T3 provide close results in general, unlike T1, we consider that the latter are sufficiently converged, and consequently adopt case T2 as our reference solution in the following.

The instantaneous stream-wise velocity field on the central $x-y$ plane at the end of the simulation is shown in Fig. 15. The velocities on the $x-z$ plane for the same time instant are shown in Fig. 16. From the plot of stream-wise velocity component $u$ in Fig 16(a), we can observe the formation of a recirculation zone just after the cylinder. Similarly, in Fig. 16(b), which shows the instantaneous cross-stream component of velocity $v$, the alternating direction of the velocity suggests the formation of a vortex trail.

While the instantaneous velocity distributions provide a qualitative idea of the flow, we are also interested in studying the statistics of the cylinder wake, which represent a more quantitative measure of the quality of the simulation. We compute the average velocity in the stream-wise and cross-stream directions on different $y-z$ sections (that is, normal to the mean flow) on the wake of the cylinder. The results in terms of averages, compared to those of [38], are shown in Fig. 17.

Observing the results, we can see that we obtain a close agreement between our results and those in the reference, although the formation of the wake is slightly delayed in our simulations when compared to the reference. This can be seen by considering that the average velocity defect on the wake starts as a deep $U$ shaped trough just behind the cylinder, where the average flow in the recirculation zone is very small or negative on average, and becomes wider and shallower (closer to the inflow velocity $U_{\infty}$ ) as the wake develops. In general, our profiles are below the expected curve, which corresponds to a larger velocity defect for a given section.

In addition, we have computed the variance of the stream-wise velocity $\left\langle u^{\prime} u^{\prime}\right\rangle$, shown in Fig. 18(a) for the near wake and in Fig. 18(b) for the far wake. The obtained variance is smaller in general than in the reference

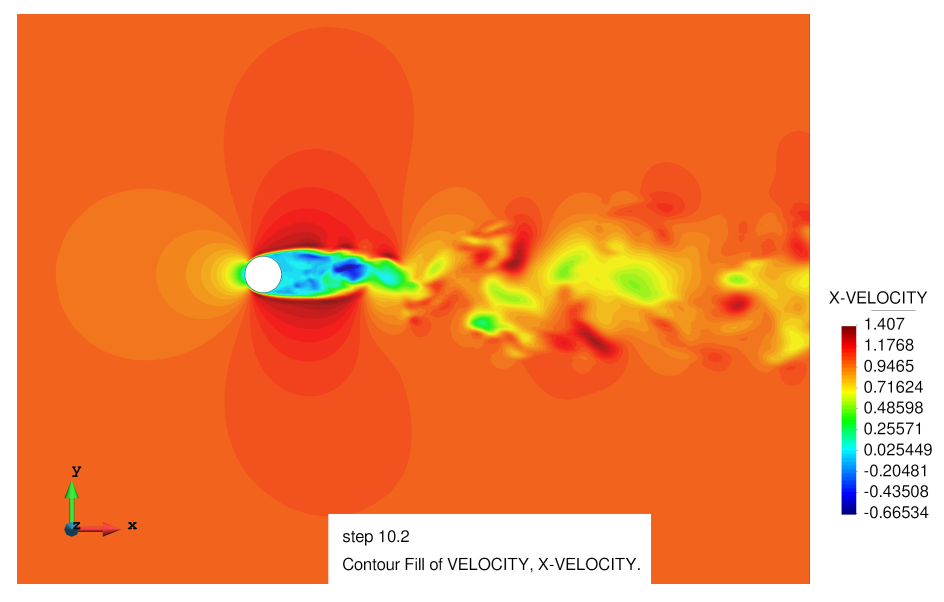

Figure 15: Flow around a cylinder - instantaneous stream-wise velocity $u$ on the $x-y$ midplane. 

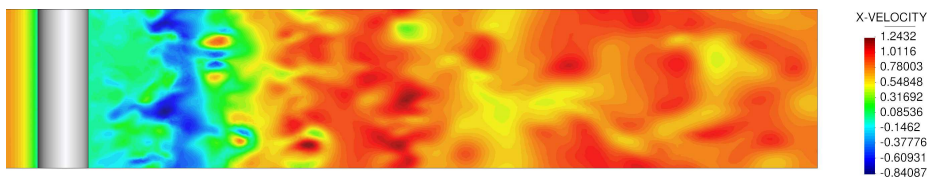

(a) Stream-wise velocity $u$.

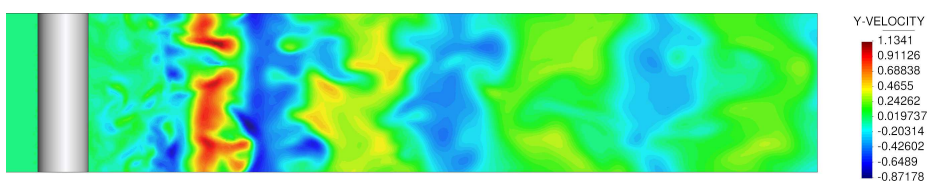

(b) Cross-stream velocity $v$.

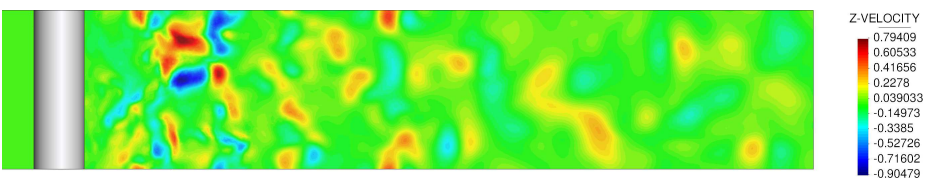

(c) Span-wise velocity $w$.

Figure 16: Flow around a cylinder - instantaneous velocities on the $x-z$ midplane.

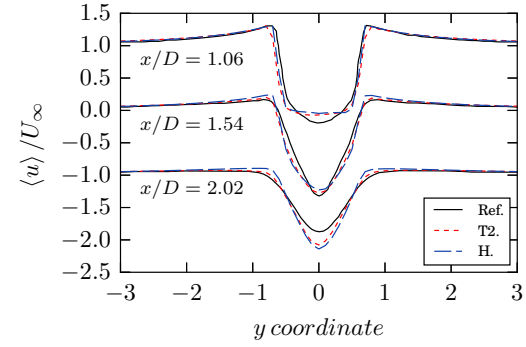

(a) Stream-wise velocity $\langle u\rangle$ (near wake).

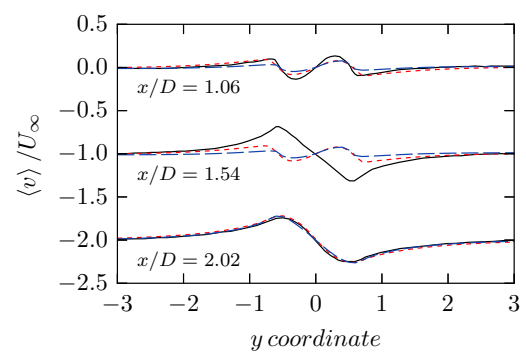

(b) Cross-stream velocity $\langle v\rangle$ (near wake).

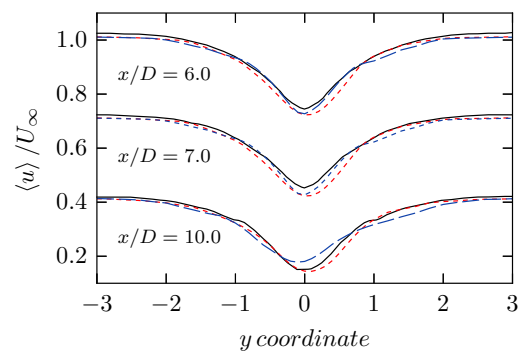

(c) Stream-wise velocity $\langle u\rangle$ (far wake).

Figure 17: Flow around a cylinder - average velocities in the wake. 


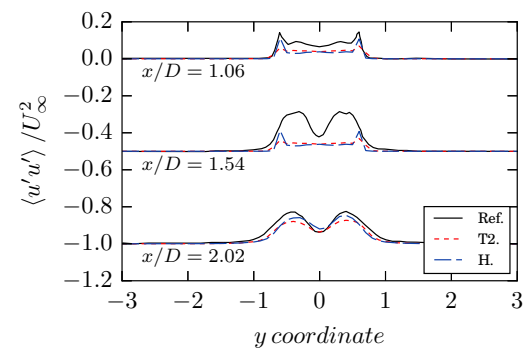

(a) $\left\langle u^{\prime} u^{\prime}\right\rangle$ correlation (near wake).

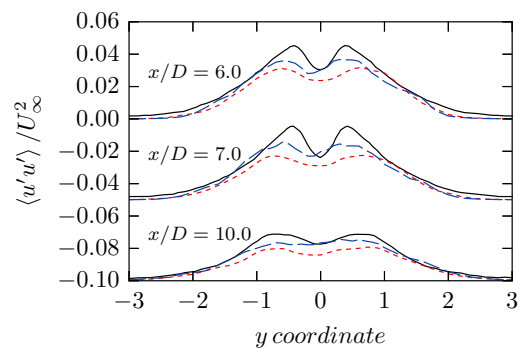

(b) $\left\langle u^{\prime} u^{\prime}\right\rangle$ correlation (far wake).

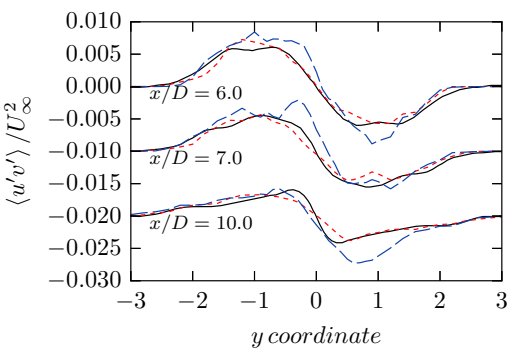

(c) $\left\langle u^{\prime} v^{\prime}\right\rangle$ correlation (far wake).

Figure 18: Flow around a cylinder - velocity correlations in the wake.

data, which suggests a smoother flow and a more diffusive solution. Finally, the cross-correlation $\left\langle u^{\prime} v^{\prime}\right\rangle$ for the far wake is shown in Fig. 18(c). This result shows some irregularity, larger in our solution than in the reference, which might be reduced with a longer simulation time.

Finally, we compute the drag coefficient $C_{D}$ and the Strouhal number St, which represents the dimensionless vortex shedding frequency, given by the expressions

$$
C_{D}=\frac{\left\langle R_{x}\right\rangle}{\frac{1}{2} \rho U_{\infty}^{2} D W} \quad \mathrm{St}=\frac{f D}{U_{\infty}}
$$

where $\left\langle R_{x}\right\rangle$ is the average force applied by the fluid on the surface of the cylinder in the stream-wise direction and $f$ is the vortex-shedding frequency on the cylinder tail, which we obtain from the lift force history $R_{y}(t)$. The results, compared to those reported in [38], are summarized in Table 2.

\begin{tabular}{c|c|c} 
Analysis & $C_{D}$ & $\mathrm{St}$ \\
\hline FIC-FEM results & 1.09 & 0.217 \\
Numerical [38] & 1.04 & 0.210 \\
Experimental (reported in [38]) & $0.99 \pm 0.05$ & $0.215 \pm 0.005$
\end{tabular}

Table 2: Flow around a cylinder - flow parameters.

The results obtained for this case show agreement to reference values in terms of the average solution in general. Variances, while in qualitative agreement with the expected results in terms of spatial distribution, tend to be underestimated. This suggests that the FIC-FEM solution has a slightly larger dissipation than required, smoothing out peaks in the fluctuating solution.

\section{Summary and conclusions}

We have introduced a new FIC-based finite element formulation for incompressible flows. Compared to previous FIC-FEM stabilized formulations of the Navier-Stokes problem [1, 17, 19, 20, 21], our method contains a new term on the momentum equation, which introduces an additional non-isotropic dissipation in the direction of the velocity gradients, and a stabilized formulation for the mass equation which is based on a second order FIC balance in space and represents an incompressible Eulerian version of the method presented for quasiincompressible flows in [2].

The method as presented has a free combination parameter $\beta_{i}$ that defines the relative weights of the classical streamline diffusion and the new gradient diffusion term in the stabilization of each component of the momentum equation. We have explored the use of a constant value for $\beta_{i}$ and also have proposed a way to define its value dynamically, based on the local features of the flow, with the intent of improving the results and reducing the dependence of the model on free parameters.

We have tested the FIC-FEM procedure on the turbulent channel flow at $\operatorname{Re}_{\tau}=395$ benchmark, where we have analyzed the performance of the different variants of the method, and the flow around a cylinder at $\operatorname{Re}=3900$. The main conclusions we extracted from the simulations can be summarized as follows:

- Hexahedral meshes provide significantly better results than tetrahedra, with $32^{3}$ hexahedra producing results of comparable quality to $6 \times 64^{3}$ tetrahedra.

- The method is quite insensitive to the free parameter $\beta$, at least for values of $\beta \geq 0.5$, for either the fixed or variable $\beta_{i}$ variants. 
- Using the variable expression for the $\beta_{i}$ coefficient results in a better approximation to the DNS curves, although it increases the non-linearity of the problem to be solved.

- The solution obtained using a variable $\beta_{i}$ coefficient presents a physically meaningful energy spectrum for modes that are well resolved with the calculation mesh.

The FIC-FEM procedure proposed has been able to produce an accurate solution for the flow examples considered. Attempting to provide a theoretical justification for this observation will be an obvious venue for further research. In this sense, it is interesting to note that the gradient diffusion terms, which constitute the main difference between the proposed formulation and other stabilization methods, has a similar structure to the family of LES models known as gradient models, such as the Clark model [40] or the Modulated Gradient Diffusion model [41]. Exploring the relation of the FIC-based stabilization procedure to such models could allow us to obtain a better understanding of the good features of the method in terms of its behavior in turbulent problems and motivate future improvements.

\section{References}

\section{References}

[1] E. Oñate, Derivation of stabilized equations for numerical solution of advective-diffusive transport and fluid flow problems, Computer Methods in Applied Mechanics and Engineering 151 (12) (1998) 233 - 265, containing papers presented at the Symposium on Advances in Computational Mechanics. doi:http://dx.doi.org/10.1016/S0045-7825(97)00119-9.

[2] E. Oñate, A. Franci, J. M. Carbonell, Lagrangian formulation for finite element analysis of quasiincompressible fluids with reduced mass losses, International Journal for Numerical Methods in Fluids 74 (10) (2014) 699-731. doi:10.1002/fld.3870.

[3] S. B. Pope, Turbulent Flows, Cambridge University Press, 2000.

[4] A. E. Tejada-Martínez, K. E. Jansen, On the interaction between dynamic model dissipation and numerical dissipation due to streamline upwind/Petrov-Galerkin stabilization, Computer Methods in Applied Mechanics and Engineering 194 (9-11) (2005) 1225 - 1248. doi:http://dx.doi.org/10.1016/j.cma.2004.06.037.

[5] A. V. Trofimova, A. E. Tejada-Martínez, K. E. Jansen, R. T. Lahey, Jr., Direct numerical simulation of turbulent channel flows using a stabilized finite element method, Computers \& Fluids 38 (4) (2009) 924 938.

[6] V. Gravemeier, M. W. Gee, M. Kronbichler, W. A. Wall, An algebraic variational multiscalemultigrid method for large eddy simulation of turbulent flow, Computer Methods in Applied Mechanics and Engineering 199 (13-16) (2010) 853 - 864, turbulence Modeling for Large Eddy Simulations. doi:http://dx.doi.org/10.1016/j.cma.2009.05.017.

[7] T. J. R. Hughes, Multiscale phenomena: Green's functions, the Dirichlet-to-Neumann formulation, subgrid scale models, bubbles and the origins of stabilized methods, Computer Methods in Applied Mechanics and Engineering 127 (1-4) (1995) 387-401. doi :DOI : 10.1016/0045-7825 (95) 00844-9.

[8] T. J. R. Hughes, G. R. Feijóo, L. Mazzei, J.-B. Quincy, The variational multiscale methoda paradigm for computational mechanics, Computer Methods in Applied Mechanics and Engineering 166 (1-2) (1998) 3-24, advances in Stabilized Methods in Computational Mechanics. doi :DOI : 10.1016/S0045-7825(98)00079-6.

[9] T. J. R. Hughes, L. Mazzei, K. E. Jansen, Large eddy simulation and the variational multiscale method, Computing and Visualization in Science 3 (2000) 47-59.

[10] R. Codina, J. Príncipe, O. Guasch, S. Badia, Time dependent subscales in the stabilized finite element approximation of incompressible flow problems, Computer Methods in Applied Mechanics and Engineering 196 (21-24) (2007) 2413-2430. doi:DOI:10.1016/j.cma.2007.01.002.

[11] O. Guasch, R. Codina, Statistical behavior of the orthogonal subgrid scale stabilization terms in the finite element large eddy simulation of turbulent flows, Computer Methods in Applied Mechanics and Engineering 261-262 (0) (2013) 154-166. doi:http://dx.doi.org/10.1016/j.cma.2013.04.006. 
[12] J. P. Boris, F. F. Grinstein, E. S. Oran, R. L. Kolbe, New insights into large eddy simulation, Fluid Dynamics Research 10 (4-6) (1992) 199.

[13] I. Akkerman, Y. Bazilevs, V. Calo, T. Hughes, S. Hulshoff, The role of continuity in residualbased variational multiscale modeling of turbulence, Computational Mechanics 41 (2008) 371-378. doi: $10.1007 / \mathrm{s} 00466-007-0193-7$.

[14] Y. Bazilevs, V. Calo, J. Cottrell, T. Hughes, A. Reali, G. Scovazzi, Variational multiscale residual-based turbulence modeling for large eddy simulation of incompressible flows, Computer Methods in Applied Mechanics and Engineering 197 (1-4) (2007) 173 - 201. doi:DOI:10.1016/j.cma.2007.07.016.

[15] M. Avila, R. Codina, J. Principe, Large eddy simulation of low mach number flows using dynamic and orthogonal subgrid scales, Computers \& Fluids 99 (2014) 44 - 66. doi:http://dx.doi.org/10.1016/j.compfluid.2014.04.003.

[16] O. Colomés, S. Badia, R. Codina, J. Príncipe, Assessment of variational multiscale models for the large eddy simulation of turbulent incompressible flows, Computer Methods in Applied Mechanics and Engineering 285 (0) (2015) 32 -63. doi:http://dx.doi.org/10.1016/j.cma.2014.10.041.

[17] E. Oñate, A stabilized finite element method for incompressible viscous flows using a finite increment calculus formulation, Computer Methods in Applied Mechanics and Engineering 182 (3-4) (2000) 355 370. doi:10.1016/S0045-7825 (99)00198-X.

[18] E. Oñate, Possibilities of finite calculus in computational mechanics, International Journal for Numerical Methods in Engineering 60 (1) (2004) 255-281. doi:10.1002/nme.961.

[19] E. Oñate, A. Valls, J. García, Computation of turbulent flows using a finite calculus-finite element formulation, International Journal for Numerical Methods in Fluids 54 (6-8) (2007) 609-637. doi:10.1002/fld.1476.

[20] E. Oñate, A. Valls, J. García, Modeling incompressible flows at low and high Reynolds numbers via a finite calculus-finite element approach, Journal of Computational Physics 224 (1) (2007) 332 - 351, special Issue Dedicated to Professor Piet Wesseling on the occasion of his retirement from Delft University of Technology. doi:http://dx.doi.org/10.1016/j.jcp.2007.02.026.

[21] E. Oñate, P. Nadukandi, S. R. Idelsohn, J. García, C. Felippa, A family of residual-based stabilized finite element methods for Stokes flows, International Journal for Numerical Methods in Fluids 65 (1-3) (2011) 106-134. doi:10.1002/fld.2468.

[22] E. Oñate, S. R. Idelsohn, C. A. Felippa, Consistent pressure Laplacian stabilization for incompressible continua via higher-order finite calculus, International Journal for Numerical Methods in Engineering 87 (15) (2011) 171-195. doi:10.1002/nme.3021.

[23] R. Codina, J. Principe, M. Ávila, Finite element approximation of turbulent thermally coupled incompressible flows with numerical sub-grid scale modeling, International Journal for Numerical Methods for Heat \& Fluid Flow 20 (2010) 492-516. doi:doi:10.1108/09615531011048213.

[24] R. Temam, Navier-Stokes Equations: Theory and Numerical Analysis, American Mathematical Soc., 2001.

[25] T. J. R. Hughes, M. Mallet, A new finite element formulation for computational fluid dynamics: III. The generalized streamline operator for multidimensional advective-diffusive systems, Computer Methods in Applied Mechanics and Engineering 58 (3) (1986) 305-328. doi :DOI:10.1016/0045-7825 (86) 90152-0.

[26] R. Codina, A discontinuity-capturing crosswind-dissipation for the finite element solution of the convectiondiffusion equation, Computer Methods in Applied Mechanics and Engineering 110 (3-4) (1993) 325 - 342. doi : 10. 1016/0045-7825 (93) 90213-H.

[27] T. J. R. Hughes, L. P. Franca, G. M. Hulbert, A new finite element formulation for computational fluid dynamics: VIII. The Galerkin/least-squares method for advective-diffusive equations, Computer Methods in Applied Mechanics and Engineering 73 (2) (1989) 173-189. doi:DOI:10.1016/0045-7825(89)90111-4.

[28] T. J. R. Hughes, The Finite Element Method: Linear Static and Dynamic Finite Element Analysis, Dover Publications, Inc, 2000.

[29] P. Dadvand, R. Rossi, M. Gil, X. Martorell, J. Cotela-Dalmau, E. Juanpere, S. R. Idelsohn, E. Oñate, Migration of a generic multi-physics framework to HPC environments, Computers \& Fluids 80 (2013) 301-309. 
[30] H. Tennekes, J. L. Lumley, A first course in turbulence, MIT Press, 1972.

[31] R. D. Moser, J. Kim, N. N. Mansour, Direct numerical simulation of turbulent channel flow up to $\operatorname{Re}_{\tau}=590$, Physics of Fluids 11 (4) (1999) 943-945. doi:10.1063/1.869966.

[32] U. Piomelli, E. Balaras, Wall-layer models for large-eddy simulations, Annual Review of Fluid Mechanics 34 (2002) 349-374. doi:\{10.1146/annurev.fluid.34.082901.144919\}.

[33] E. Bou-Zeid, C. Meneveau, M. Parlange, A scale-dependent Lagrangian dynamic model for large eddy simulation of complex turbulent flows, Physics of Fluids (1994-present) 17 (2) (2005) 025105. doi:http://dx.doi.org/10.1063/1.1839152.

[34] F. E. Ham, F. Lien, A. B. Strong, A fully conservative second-order finite difference scheme for incompressible flow on nonuniform grids, Journal of Computational Physics 177 (1) (2002) 117 - 133. doi:http://dx.doi.org/10.1006/jcph.2002.7006.

[35] C. Norberg, Fluctuating lift on a circular cylinder: review and new measurements, Journal of Fluids and Structures 17 (1) (2003) 57-96. doi:10.1016/S0889-9746(02)00099-3.

[36] J. H. Lienhard, Synopsis of lift, drag, and vortex frequency data for rigid circular cylinders, Tech. rep., Washington State University (1966).

[37] L. Ong, J. Wallace, The velocity field of the turbulent very near wake of a circular cylinder, Experiments in Fluids 20 (1996) 441-453, 10.1007/BF00189383.

[38] A. G. Kravchenko, P. Moin, Numerical studies of flow over a circular cylinder at $R e_{D}=3900$, Physics of Fluids 12 (2) (2000) 403-417.

[39] P. Beaudan, P. Moin, Numerical experiments on the flow past a circular cylinder at sub-critical Reynolds number, Tech. Rep. TF-62, Department of Mechanical Engineering, Stanford University (December 1994).

[40] R. A. Clark, J. H. Ferziger, W. C. Reynolds, Evaluation of subgrid-scale models using an accurately simulated turbulent flow, Journal of Fluid Mechanics 91 (1979) 1-16. doi:10.1017/S002211207900001X.

[41] H. Lu, F. Porté-Agel, A modulated gradient model for large-eddy simulation: Application to a neutral atmospheric boundary layer, Physics of Fluids 22 (1). doi:http://dx.doi.org/10.1063/1.3291073. 